\title{
Consecuencias De Ser Padre A Temprana Edad Sobre Los Ingresos: Caso Colombiano
}

\author{
Christian Camilo Gómez ${ }^{1}$ \\ Resumen
}

Este estudio estima la penalidad que se presenta en los ingresos laborales de los padres que tuvieron sus hijos a edad temprana, antes de los 21 años, en relación a aquellos que postergan su paternidad. Para corregir los problemas de endogeneidad, se usan técnicas de correspondencia (propensity score matching) y método de Heckman. Los resultados sugieren que los padres a edad temprana tienen una penalidad del $11,7 \%$ en sus ingresos por hora. Los impactos son mayores en magnitud para las mujeres $(12,6 \%)$ que para los hombres $(5,1 \%)$ y los canales de transmisión son heterogéneos según sexo. La paternidad temprana se produce en un momento crítico del ciclo de vida, donde aumenta la probabilidad de interrumpir eventos importantes en la formación de capital humano, y produce cambios en la forma en que los padres jóvenes se vinculan al mercado laboral como consecuencia del cuidado y mantenimiento económico del niño.

Palabras clave: salarios, diferencias salariales, maternidad temprana, "birth timing", Colombia, propensity score.

Códigos JEL: J13, J16, J24, I30.

\footnotetext{
${ }^{1}$ cc.gomez88@uniandes.edu.co. El autor quiere agradecer el apoyo y recomendaciones de Ximena Peña. A su vez, quiere gratificar los comentarios de Carlos Ospino, Piedad Urdinola y todo el equipo del seminario de investigación de la profesora Ximena Peña (Rodrigo Galindo, Camila Uribe, Ricardo Salas, Thibaud Deguilhem.)
} 


\section{Introducción}

Aunque en Colombia ha disminuido significativamente el número de hijos, y las tasas globales de fecundidad están en niveles comparables con las de países desarrollados (Flórez, 2000), la edad promedio de concepción del primer hijo es bastante más baja en Colombia que en los países desarrollados.

En el año 2000 la edad media para tener hijos en la Unión Europea era de $29.52 .^{2}$ Según datos de Eurostat, en 2010, en Suiza la edad media de las mujeres para tener su primer hijo se situó en 31.7 años, en España 31.3, en Alemania 30.0, en Austria 28.5 y en Italia 31.0. Desde mediados de los años setenta, esta situación destaca una tendencia hacia una maternidad cada vez más tardía. Este escenario para Centro América y países como Ecuador, República Dominicana y Colombia, resulta bastante distante. Según datos del $\mathrm{DANE}^{3}$, se estima que para el caso colombiano las personas en promedio tienen su primer hijo entre los 18 y 21 años. ${ }^{4}$ Los resultados del tercer seguimiento de la encuesta longitudinal de Familias en Acción ${ }^{5}$ (ELFA) muestran que el 76,1\% de las mujeres entrevistadas reportaron haber tenido su primer hijo antes de los 20 años.

De igual forma, el país ha convivido con un significativo incremento en las tasas de fecundidad adolecente. Para el año 2000, Flórez y Soto (2007) estimaban que la cifra de mujeres entre 15 y 19 años que habían experimentado un embarazo ascendía a 19,1\%. Según datos de Profamilia se estimó que esta cifra para el 2005 llegaba al 31,5\% para la población más pobre (Silva A., González P. \& Torres M., 2008). Este creciente fenómeno estaría, en algún grado, relacionado con reducciones del promedio de la edad de procreación inicial.

La maternidad temprana es actualmente fuente de gran preocupación desde la política pública, porque tradicionalmente se ha asociado con pérdidas en el bienestar de los padres

\footnotetext{
${ }^{2}$ Media calculada con los 11 países de los que hay información. 3 Departamento Administrativo Nacional de Estadística (DANE).

${ }^{4}$ No existe en las estadísticas colombianas un dato exacto sobre procreación inicial. Los datos a los que hace referencia el párrafo se encuentran en la gráfica 1.1 del Apéndice 1, y usa como aproximación la edad de aquellos padres que lo fueron por primera vez.

5 Familias en acción se refiere a un programa de transferencias condicionas del gobierno colombiano, por lo que la población objetivo de la encuesta (ELFA) es población en situación de vulnerabilidad.
} 
jóvenes, se muestra como potenciador de círculos de pobreza y deterioro social. Denise y Pol (1982) afirman que ésta puede tener implicaciones sociales y sicológicas más allá de la misma maternidad. Resumen el impacto adverso de la maternidad temprana en menos educación, alta fecundidad posterior, baja participación laboral, ingresos reducidos, estrés económico durante la vida y oportunidades limitadas.

Este trabajo busca identificar las consecuencias económicas del bajo promedio de procreación inicial del país, en particular, si existe una penalidad sobre los ingresos laborales de los padres que tuvieron sus hijos a edad temprana, en relación a aquellos que postergan su paternidad. Se analiza qué proporción del impacto se explica por ser padre adolescente, y qué otra por el bajo promedio de edad para la concepción del primer hijo. Este abordaje, determina la importancia del fenómeno, y establece que es necesario redimensionar la perspectiva que se tiene sobre la problemática del embarazo adolescente. El estudio también indaga sobre los canales y formas a través de los cuales ese impacto es explicado.

Se han realizado estudios en el país que evidencian que la maternidad disminuye la participación laboral y los ingresos de las mujeres. También se ha explorado el posible impacto en los años de educación y la influencia de la fecundidad en la generación de trampas de pobreza. Sin embargo, en Colombia no se conocen estudios de los efectos sobre los ingresos laborales de las madres jóvenes, gran parte de las investigaciones disponibles se han concentrado en el embarazo adolecente; no se ha profundizado entonces, si el impacto negativo de la maternidad adolecente se puede extender a edades más allá de la adolescencia, o cuáles son los inconvenientes de que la edad media de procreación inicial sea bajo. Tampoco se han estimado las posibles implicaciones de la paternidad temprana. Este trabajo mide las consecuencias de ser padre a edad temprana sobre la oferta laboral de los individuos en el mediano plazo, tanto para hombres como para mujeres. Explorando esto, se propone promover estudios de política pública para elevar el promedio de edad en la cual los colombianos tienen sus hijos.

El presente trabajo define la paternidad temprana como aquella que se presenta antes de los 21 años de edad, determinado porque es hacia esta edad donde se concentra el promedio de 
procreación inicial en el país, y además, porque el Ministerio de Educación Nacional de Colombia fija esta edad como la edad media para la finalización de la educación superior.

Tomando como base la Gran Encuesta Integrada de Hogares, se usan derivaciones de la ecuación salarial de Mincer (1974) y corrección del sesgo de selección de Heckman (1979) para estimar el impacto de la maternidad/paternidad temprana sobre los ingresos. Estas estimaciones se complementan con técnicas de correspondencia (Propensity Score Matching) para corregir la posible endogeneidad de ser padre joven.

El trabajo se concentra en los efectos en el mediano plazo, para lo cual se realizan diversas especificaciones para una población entre 25 y 35 años.

Los resultados sugieren que existe una penalidad sobre los ingresos, adicional a la de ser padre, causada por el evento de ser padre a edad temprana. A pesar de incluir diferentes controles sociodemográficos y aplicar las técnicas de correspondencia, los padres jóvenes ganan en promedio $11,7 \%$ menos que sus pares que postergaron la paternidad. Los resultados muestran que al parecer la acumulación de capital humano es un canal fundamental, pero no es el único canal a través del cual esta penalidad es explicada. La forma en que los padres se vinculan al mercado laboral también se ve afectada por el hecho de ser padre joven. Las magnitudes del impacto y los canales son diferentes para hombres y mujeres.

Estos resultados no solo dependen de ser padre adolescente: excluyendo los eventos de fecundidad adolescente, se encontró una penalidad del 9,1\% en los ingresos de los padres que lo fueron entre los 18 y 21 años, en comparación con aquellos que fueron padres después de esa edad.

El presente artículo está organizado de la siguiente manera. La segunda sección muestra las principales contribuciones sobre el tema que se han forjado en la literatura internacional, latino americana y en Colombia. La sección 3 describe los datos a utilizar, junto con algunas estadísticas descriptivas y en la sección 4 se especifica la metodología seguida por el autor y las estrategias de identificación. En la sección 5 se presentan los principales resultados. La sección 6 presenta las conclusiones y recomendaciones del estudio. 


\section{Revisión de la literatura}

Gran parte la literatura sobre fecundidad se fundamenta en la investigación demográfica y sociológica. Existe un amplio acervo de literatura sobre las causas y consecuencias económicas de la maternidad. Los estudios que iniciaron este análisis estuvieron impulsados primordialmente por la teoría neoclásica, teniendo como precursor principal a Becker (1960) quien estudió las adversidades socioeconómicas y de salubridad que generaba tener un mayor número de hijos. Es en estudios como el de Mincer (1974) y el mismo Becker (1985) donde se dan las primeras fundamentaciones sobre el impacto de la maternidad en los ingresos, sugiriendo los efectos negativos que enfrentan las madres sobre su experiencia laboral y su ingreso, dado el tiempo que demanda la crianza de sus hijos.

La literatura sobre la maternidad temprana ha estado tradicionalmente ligada al tema de la fecundidad adolecente, descrita como un determinante fuerte de la persistencia de pobreza intergeneracional. A la maternidad adolescente se le adjudica la responsabilidad de reducciones sustanciales de los años de educación formal y de generar cambios importantes en las características de la oferta laboral de las madres adolescentes, cuando se compara con las mujeres que postergan su maternidad (Trussell J., 1980; Jencks, 1989; Geronimus \& Korenman, 1992; Klepinger et al., 1997; Taniguchi, 1999; Chevalier \& Viitanen, 2001).

Desde hace algunas décadas varias investigaciones han nutrido ésta literatura sobre todo en países desarrollados. Las consecuencias que la maternidad adolescente trae al bienestar económico de los progenitores no se consideran exógenas, y están correlacionadas con diversas características intrínsecas de los padres jóvenes (Geronimus y Korenman, 1992; Klepinger et al., 1997). Los primeros presentaron una estimación teniendo en cuenta las heterogeneidades de las características familiares comparando hermanas que tuvieron sus hijos a diferentes edades, demostrando que las mediciones previas que se habían realizado

en Estados Unidos contenían sesgos y sobre estimaban las consecuencias de la maternidad adolescente. 
Desde entonces, los esfuerzos se han concentrado en utilizar técnicas metodológicas que ayuden a corregir los sesgos que la estimación de la maternidad adolescente trae sobre diferentes resultados. Chevalier (2002) ${ }^{6}$ encuentra que aunque se reduce el efecto negativo en educación, enrolamiento e ingreso laboral, este se mantiene, aun cuando se tienen en cuenta las características heterogéneas (no observables) de las mujeres que tuvieron un hijo en la adolescencia. Además, en los casos en que la situación económica sea desfavorable, puede ser indispensable comenzar a realizar actividades generadoras de ingreso que permitan el mantenimiento de un hijo (Alcázar, 2006).

Algunas investigaciones explotan la información de los abortos no inducidos, un experimento natural, y concluyen que los efectos de la maternidad temprana eran más pequeños de lo que se había considerado en estudios previos (Hotz, 1995; Hotz et al., 2005). Estos estudios encuentran, sin embargo, que las madres adolescentes trabajaban menos horas y tenían ingresos laborales más bajos.

Desde hace poco más de una década en los países de América Latina se ha comenzado a explorar los impactos de la maternidad adolecente en el desarrollo socio económico de las madres (Alcázar, 2006 para Peru; Gerstenbluth et al., 2009 para Uruguay; Berthelon et al., 2010 para Chile). En general, se han evaluado las probabilidades de alcanzar logros educativos o completar los estudios. Alcázar (2006) muestra como en Perú las diferencias en acumulación de capital humano de las madres adolescentes parecieran manifestarse a mediano plazo, y no en gran medida, en el acceso al empleo, sino más bien en las condiciones laborales a las que se ven sometidas las madres adolescentes.

Acevedo et al. (2012) siguen a Holtz et al. (1995) para evaluar la acumulación de capital humano y participación laboral de las madres adolescentes en México, llegando a conclusiones poco intuitivas, encuentran que las mujeres que dieron a luz durante su adolescencia tienen en promedio 0.34 más años de educación y tiene una probabilidad de 21 puntos porcentuales más alta de estar empleadas.

${ }^{6}$ Chevalier (2002) Es un estudio para Inglaterra. 
Para el caso colombiano se tienen algunos estudios acerca de los impactos de la maternidad adolescente que se han concentrado particularmente en el impacto en la acumulación de capital humano. Núñez y Cuesta (2006) y Núñez (2008) usando variables instrumentales, estiman en 2 años el impacto del embarazo adolecente en el promedio de educación de las mujeres. Además, muestran que la fecundidad adolecente tiene efectos negativos sobre la participación laboral, la estructura y el tamaño del hogar. Por otra parte, Urdinola y Ospino (2013), a través de un pseudo panel, concluyen que para Colombia no existen efectos de largo plazo en la educación o tasas de ocupación en las madres adolescentes.

Con respecto a la penalidad sobre los ingresos, Olarte y Peña (2010) confirman la existencia de una penalización significativa de la maternidad sobre los ingresos de las mujeres, que se agravaba cuando el hijo es altamente dependiente. Sugieren que pareciera haber un impacto en los ingresos de las madres jóvenes pero no ahondan en este análisis. ${ }^{7}$

En lo que respecta a la paternidad, la literatura no ha presentado el mismo volumen de información como en el tema de las madres. Maureen (1996) concluye que en Estados Unidos los padres adolecentes completan menos años de educación y son más propensos a no finalizar la secundaria, entran antes al mercado laboral, pero cuando se encuentran entre los 20 y 30 años ganan menos salario.

Este trabajo contribuye en varias dimensiones a la literatura sobre el tema. Primero, para el caso colombiano no existe un análisis que aborde las implicaciones que tiene el bajo promedio de procreación inicial de los colombianos sobre oferta laboral de los individuos. Los trabajos existentes tratan sobre embarazo adolescente únicamente en mujeres, y no se profundiza en las características de la vinculación laboral o los ingresos laborales de los padres jóvenes. Segundo, se analizan los posibles efectos de la paternidad temprana cuyo caso nunca se ha estudiado en el país. Tercero, identifica (hasta donde la estructura de los datos lo permite) los canales a través de los cuales se generan los impactos negativos sobre los ingresos. En particular, encontramos que el abandono de estudios y los cambios en la

\footnotetext{
${ }^{7}$ Gamboa y Zuluaga (2013) por el contrario, no encontraron evidencia de brechas salariales significativas entre madres y no madres, cuando vinculan variables de educación al modelo de descomposición de Ñopo (2008). Esto puede estar explicado porque la educación es una variable altamente influenciada por el hecho de ser madre y no una característica pre-maternidad.
} 
forma en que los padres jóvenes se insertan al mercado laboral, son los principales determinantes. Finalmente, se exploran las implicaciones de política pública de los resultados.

\section{Datos}

Los datos que se van a utilizar principalmente para efectuar las estimaciones y el análisis comprenden un año de acumulación de cortes transversales de la Gran Encuesta Integrada de Hogares (GEIH), desde octubre de 2012 hasta septiembre de 2013.

Este periodo fue seleccionado dado que desde esta fecha se incorporó a la GEIH un módulo sobre fecundidad que se aplica a hombres entre los 14 y 60 años y a mujeres entre 12 y 55 años. Esta información se complementa con una amplia batería de preguntas sobre mercado laboral, características individuales y de hogar. Además, actualmente es la única fuente de información (a través de encuesta) sobre fecundidad de hombres en Colombia.

La encuesta no presenta variaciones metodológicas durante este periodo. La GEIH es recolectada por el DANE, se trata de una encuesta continua que se realiza desde el año 2006. Esta base de datos provee un muestra aleatorizada y estratificada de 66.000 hogares aproximadamente (al trimestre) que se traducen en cerca de 264.000 individuos que se entrevistan de manera directa. Es representativa a nivel nacional, por dominios: cabecera, resto $^{8}$ y 24 ciudades principales. $^{9}$

Adicionalmente, se utilizó información de registros administrativos sobre nacimientos provenientes de la operación Estadísticas Vitales (EV) del DANE y Ministerio de Protección Social (MPS), desde el año 1998 hasta el año 2012, y la información del Registro Civil de Nacimiento de la Registraduria Nacional para los años de 1990 a $1998 .{ }^{10}$

Para los efectos de este estudio se selecciona una muestra de padres entre los 25 y 35 años de edad, con el objetivo de impedir que se hagan comparaciones intergeneracionales, es decir, solo se evaluará una cohorte poblacional donde no hay padres e hijos en la misma

\footnotetext{
${ }^{8}$ Cabecera se refiere a todo centro poblado con alcaldía y Resto se refiere a los demás centros poblados y rural disperso.

${ }^{9}$ Los resultados obtenidos a través de esta encuesta son generalizables a toda la población, exceptuando los antiguos territorios nacionales, donde reside un poco menos del 5\% del total de la población colombiana.

${ }^{10}$ Estas consultas apoyaron metodológicamente el propensity score que se detalla en la metodología.
} 
muestra, evitando el posible sesgo de comparar generaciones distintas. Por otra parte, esta selección nos ayuda a concentrarnos en los efectos en el mediano plazo de ser padre joven. Evaluar que los impactos sean heterogéneos a través tiempo haría parte de una nueva investigación.

Partiendo de esta población se clasificó a la población padre joven cuando manifestaron haber tenido hijos antes de los 21 años. La selección de esta edad surge por dos motivos, el primero, porque es la edad promedio en la que los colombianos tienen a sus primogénitos, y segundo, por las consideraciones del Ministerio de Educación Nacional, en donde se determina que, en un escenario sin perturbaciones, un colombiano a los 21 años debió haber culminado la educación superior.

El análisis a su vez se centrará en población no migrante por dos razones. Primero, porque es indispensable para el funcionamiento técnico de la predicción de ser padre joven (se detalla en la metodología). Segundo, el objeto del estudio es identificar una penalidad en los ingresos, y realizar esta elección en la muestra nos permitirá seleccionar individuos más homogéneos, evitando la contaminación de los resultados con sesgos en los ingresos provenientes de migraciones laborales y/o de discriminación.

El cuadro 1 presenta la selección de la muestra. La muestra comprendida de la población entre 25 y 35 años contiene 133.056 observaciones que representan 7’578.330 colombianos, cerca del $60 \%$ de esta población no ha presentado cambios migratorios, 50.551 observaciones corresponden a individuos que reportaron haber sido padres, de los cuales el $73 \%$ reportaron ingresos coherentes. ${ }^{11}$

Cuadro 1. Composición de la muestra (Octubre 2012 - Septiembre 2013)

\begin{tabular}{|c|c|c|c|c|c|c|}
\hline & \multicolumn{3}{|c|}{ No. de observaciones } & \multicolumn{3}{|c|}{$\begin{array}{l}\text { No. de observaciones aplicando factor de } \\
\text { expansión }\end{array}$} \\
\hline & Total & Hombres & Mujeres & Total & Hombres & Mujeres \\
\hline $\begin{array}{l}\text { Población objetivo del módulo de Fecundidad - } \\
\text { GEIH }\end{array}$ & 516.211 & 239.743 & 276.468 & 29.159 .664 & 14.353 .078 & 14.806 .586 \\
\hline Población del módulo de 25 a 35 años & 133.056 & 61.335 & 71.721 & 7.578 .330 & 3.691 .121 & 3.887 .209 \\
\hline No han presentado cambios migratorios & 74.308 & 34.567 & 39.741 & 4.063 .470 & 2.007 .188 & 2.056 .282 \\
\hline Reportaron haber sido padres & 50.551 & 20.136 & 30.415 & 2.839 .434 & 1.199 .920 & 1.639 .514 \\
\hline Cuyo ingreso reportado es coherente & 37.020 & 18.531 & 18.489 & 2.102 .491 & 1.122 .613 & 979.878 \\
\hline
\end{tabular}

Fuente: DANE - GEIH, cálculos propios.

\footnotetext{
${ }^{11}$ Ingreso coherente: Son los ingresos que resultan luego de eliminar las observaciones que reportan ingresos inferiores a un dólar por día al mes y que pertenecen a estratos superiores y las correspondientes a individuos que trabajan más de 40 horas por semana y pertenecen a estratos superiores al tres que reportan ingresos mensuales inferiores a un salario mínimo de 2013.
} 
Ahora, analizando los datos de la GEIH se presentan a continuación, descripciones sobre la composición de la población objetivo, en términos de la incidencia de la maternidad/paternidad joven según edades y sexo, junto con una caracterización del nivel educativo, composición del hogar y los ingresos laborales de la población padre joven.

El 50,1\% de los jóvenes de 25 años son padres, para las personas de 35 años este porcentaje asciende al 81,3\%. Para el periodo de estudio, en promedio, más de la mitad (53\%) de los padres que tienen entre 25 y 35 años han sido padres a edad temprana.

La incidencia de la paternidad temprana (que se sigue de la definición de este documento), es diferente cuando se analiza por grupo etario y sexo. La ocurrencia de la fecundidad antes de los 21 años es más prominente en mujeres. El gráfico 1 muestra cómo en Colombia la proporción de padres que lo son a edad temprana, es mayor para personas de 25 años. Para el caso de los hombres, el 29,9\% de los padres de 35 años lo fueron a temprana edad, mientras que para los padres de 25 años esta misma proporción es de 49,9\%. La situación es bastante similar para las mujeres. Sin embargo, los niveles son superiores comparados a los de los hombres: 58,2\% de las madres de 35 años lo fueron a temprana edad, ascendiendo a 76,4\% en las madres de 25 años.

\section{Gráfica 1. Porcentaje de padres jóvenes en el total de padres por sexo y edad Total Nacional - datos expandidos ${ }^{12}$ \\ (Octubre 2012 - Septiembre 2013) \\ Mujeres}

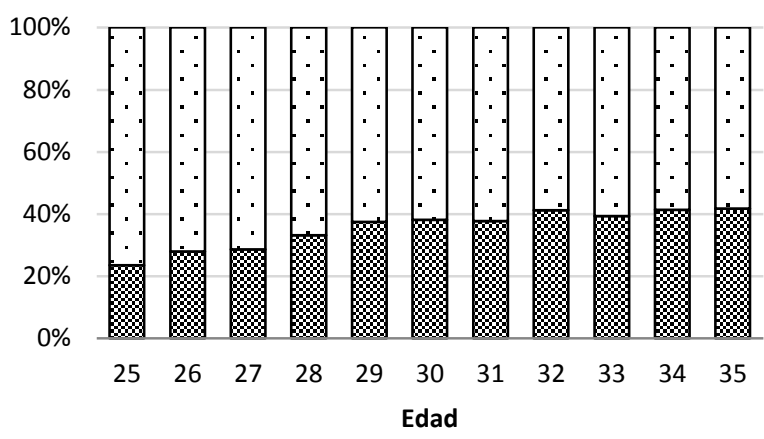

圆 No padres a edad temprana

$\square$ Padres a edad temprana

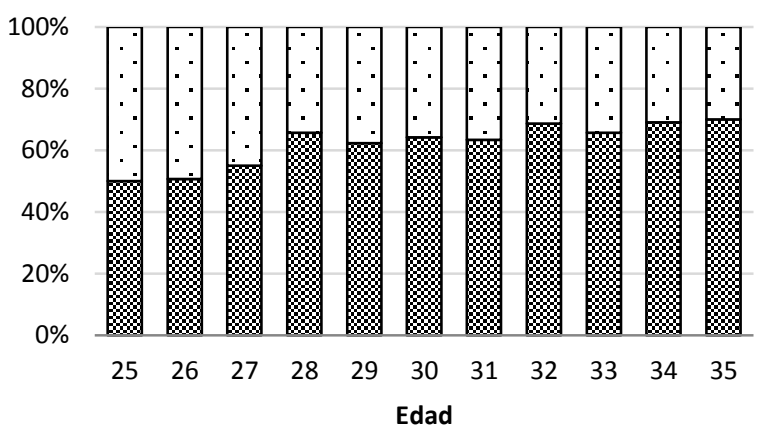

№ padres a edad temprana

\footnotetext{
${ }^{12}$ Esta información es calculada para la población objetivo del estudio, es decir, padres entre 25 y 35 años no migrantes. El factor de expansión es recalibrado para ser representativo de nueve meses. Fuente: DANE - Cálculos propios
} 
Gran parte de la población de padres objeto de estudio $(34,5 \%)$ cuenta con secundaria completa. Para este nivel educativo, la gráfica 2 muestra que cerca de la mitad de esta población fue padre a edad temprana.

Una mayor prevalencia de la paternidad/maternidad joven, se evidencia a niveles educativos inferiores, esto puede asociarse como causa y/o consecuencia de la misma. Para los niveles educativos superior incompleta y superior completa la proporción de padres jóvenes es de $42,3 \%$ y $24,3 \%$ respectivamente.

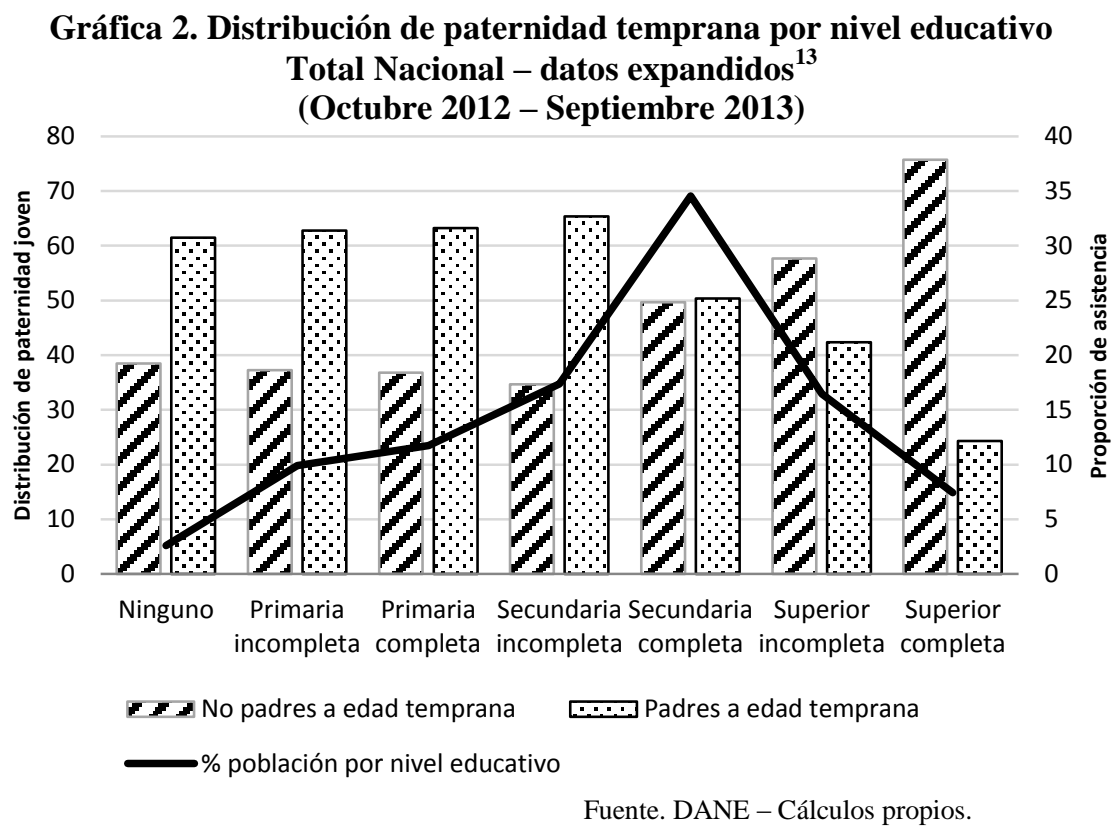

El Cuadro 2 presenta estadísticas descriptivas desagregadas según el momento de la paternidad/maternidad. Los padres jóvenes son menores en edad, en su mayoría mujeres. Tienen menos años de escolaridad y alcanzan menos logros educativos. El capital humano acumulado es más bajo para las madres jóvenes que para los padres jóvenes. Los hombres que fueron padres a edad temprana viven menos en pareja y las mujeres son más solteras. Para ambos sexos, el número de hijos es mayor para los padres jóvenes.

Con respecto a los ingresos laborales de los padres, el cuadro 3 ilustra diferencias, que resultaron ser estadísticamente significativas, en los ingresos de las personas entre 25 y 35 años, según el momento de su paternidad. Existe una razón de 1,22 entre los salarios de los

\footnotetext{
${ }^{13}$ Esta información es calculada para la población objetivo del estudio, es decir, padres entre 25 y 35 años no migrantes. El factor de expansión es recalibrado para ser representativo de doce meses. El eje secundario aplica para el porcentaje de población por nivel educativo.
} 
padres que no fueron padres a temprana edad frente a sus pares que si lo fueron. Para el caso de las mujeres esta razón alcanza 1,96. En otras palabras, las madres a edad temprana reportan un ingreso $50 \%$ menor en relación aquellas que postergaron su maternidad. La gráfica 1.3 del apéndice 1, evidencia que cuando se es padre joven existe cierto desplazamiento a la izquierda de la distribución del logaritmo del ingreso, especialmente para mujeres. Gran parte de las diferencias que se encuentran descriptivamente en los ingresos laborales, contienen sesgos explicados por diversas variables y condiciones socioeconómicas, que son distintas entre padres jóvenes y padres no jóvenes, por lo que es necesario controlar esta heterogeneidad. Asunto que se tratará en la metodología.

Cuadro 2. Estadísticas descriptivas de la población padre de 25 a 35 años, no migrante.

\begin{tabular}{|c|c|c|c|c|c|c|c|c|c|}
\hline & \multicolumn{3}{|c|}{ Total } & \multicolumn{3}{|c|}{ Mujeres } & \multicolumn{3}{|c|}{ Hombres } \\
\hline & $\begin{array}{c}\text { No padres a } \\
\text { edad } \\
\text { temprana } \\
\end{array}$ & $\begin{array}{c}\text { Padres a } \\
\text { edad } \\
\text { temprana }\end{array}$ & $\begin{array}{l}\text { Diferencia } \\
\text { de medias } \\
\text { (t-test) }\end{array}$ & $\begin{array}{c}\text { No padres a } \\
\text { edad } \\
\text { temprana }\end{array}$ & $\begin{array}{c}\text { Padres a } \\
\text { edad } \\
\text { temprana }\end{array}$ & $\begin{array}{l}\text { Diferencia } \\
\text { de medias } \\
\text { (t-test) }\end{array}$ & $\begin{array}{c}\text { No padres a } \\
\text { edad } \\
\text { temprana }\end{array}$ & $\begin{array}{c}\text { Padres a } \\
\text { edad } \\
\text { temprana }\end{array}$ & $\begin{array}{c}\text { Diferencia } \\
\text { de medias } \\
\text { (t-test) }\end{array}$ \\
\hline Sexo (Mujer) & 0.480 & 0.720 & $-0.240 * * *$ & & & & & & \\
\hline Edad & 30.541 & 29.880 & $0.661 * * *$ & 30.479 & 29.869 & $0.610 * * *$ & 30.599 & 29.907 & $0.691 * * *$ \\
\hline \multicolumn{10}{|l|}{ Educación } \\
\hline Años de escolaridad & 11.084 & 9.286 & $1.798 * * *$ & 11.947 & 9.429 & $2.518 * * *$ & 10.286 & 8.918 & $1.368^{* * * *}$ \\
\hline $\begin{array}{l}\text { Bachiller } \\
\text { Técnico o }\end{array}$ & 0.432 & 0.403 & $0.029 * * *$ & 0.424 & 0.406 & $0.018 * * *$ & 0.439 & 0.394 & $0.045^{* * *}$ \\
\hline tecnológico & 0.173 & 0.111 & $0.062 * * *$ & 0.219 & 0.123 & $0.096^{* * * *}$ & 0.130 & 0.078 & $0.052 * * *$ \\
\hline Universitario & 0.123 & 0.036 & $0.087 * * *$ & 0.163 & 0.038 & $0.125^{* * *}$ & 0.085 & 0.028 & $0.057 * * *$ \\
\hline Posgrado & 0.024 & 0.005 & $0.019 * * *$ & 0.032 & 0.005 & $0.027 * * *$ & 0.017 & 0.006 & $0.011^{* * *}$ \\
\hline \multicolumn{10}{|l|}{$\begin{array}{l}\text { Estructura del } \\
\text { hogar }\end{array}$} \\
\hline Jefe de hogar & 0.387 & 0.339 & $0.047 * * *$ & 0.182 & 0.242 & $-0.060 * * *$ & 0.575 & 0.590 & $-0.015^{* *}$ \\
\hline Soltero & 0.113 & 0.097 & $0.016^{* * *}$ & 0.127 & 0.094 & $0.033 * * *$ & 0.101 & 0.105 & -0.004 \\
\hline Cohabita & 0.741 & 0.701 & $0.040 * * *$ & 0.680 & 0.672 & 0.009 & 0.797 & 0.778 & $0.020 * * *$ \\
\hline \multicolumn{10}{|l|}{ Hijos } \\
\hline Número de hijos & 1.511 & 2.300 & $-0.788 * * *$ & 1.462 & 2.314 & $-0.852 * * *$ & 1.557 & 2.264 & $-0.707 * * *$ \\
\hline Un hijo & 0.617 & 0.251 & $0.366^{* * *}$ & 0.643 & 0.242 & $0.401 * * *$ & 0.593 & 0.272 & $0.320 * * *$ \\
\hline Dos hijos & 0.291 & 0.401 & $-0.110^{* * *}$ & 0.283 & 0.404 & $-0.121 * * *$ & 0.299 & 0.395 & $-0.096^{* * *}$ \\
\hline Más de tres hijos & 0.092 & 0.348 & $-0.256^{* * * *}$ & 0.074 & 0.354 & $-0.280 * * *$ & 0.109 & 0.332 & $-0.224 * * *$ \\
\hline Observaciones & 24916 & 25635 & & 11962 & 18453 & & 12954 & 7182 & \\
\hline
\end{tabular}


Cuadro 3. Media de ingresos laborales de personas entre 25 y 35 años.

\begin{tabular}{|c|c|c|c|c|c|c|}
\hline & & Mujeres & & & Hombres & \\
\hline & $\begin{array}{c}\text { No padres a } \\
\text { edad temprana }\end{array}$ & $\begin{array}{c}\text { Padres a edad } \\
\text { temprana }\end{array}$ & $\begin{array}{l}\text { Diferencia de } \\
\text { medias (t-test) }\end{array}$ & $\begin{array}{c}\text { No padres a } \\
\text { edad temprana }\end{array}$ & $\begin{array}{c}\text { Padres a edad } \\
\text { temprana }\end{array}$ & $\begin{array}{l}\text { Diferencia de } \\
\text { medias (t-test) }\end{array}$ \\
\hline Ingreso laboral & COP\$ 1032577 & COP\$ 524345 & $* * *$ & COP\$ 830048 & COP\$ 678226 & $* * *$ \\
\hline $\begin{array}{l}\text { Logaritmo ingreso } \\
\text { laboral (monetario) } \\
\text { Logaritmo del ingreso } \\
\text { laboral por hora } \\
\text { (monetario) }\end{array}$ & 13.109 & 12.718 & $* * *$ & 13.368 & 13.257 & $* * *$ \\
\hline
\end{tabular}

\section{Metodología}

\subsection{Reducción del sesgo de las estimaciones}

La maternidad, y en particular la maternidad adolescente, está correlacionada con diversos resultados socio-económicos (Geronimus, 1992; Klepinger et al., 1997; Chevalier, 2002; Hotz, 2005), como por ejemplo el ingreso laboral, que es la variable de interés en este estudio. Por tanto, la endogeneidad puede ser un problema en la estimación. El coeficiente estimado de la maternidad temprana en la ecuación de Mincer estará probablemente sesgado, debido a que la penalidad del ingreso puede estar explicada por variables no observables y características pre-existentes, entre aquellos padres que tuvieron sus hijos antes de los 21 años y aquellos que postergaron su maternidad, y no porque exista una relación causal entre la maternidad/paternidad temprana con resultados adversos en el mediano plazo. Como la decisión de fecundidad puede ser simultánea, por ejemplo, a la decisión de educarse, el coeficiente estimado del indicador de padre joven con cierta probabilidad esta correlacionado con el término del error, violando uno de los supuestos básicos del método de regresión por mínimos cuadrados ordinarios.

Intuitivamente, se podría pensar que las madres o padres jóvenes tienen características individuales, contextuales y socioeconómicas, que establecen diferencias salariales persistentes entre estos y aquellos que postergan su maternidad, a pesar de que no tengan 
hijos a temprana edad y de esta manera se sobreestimaría el efecto de la maternidad o paternidad en el logaritmo del ingreso u otras variables de resultado. ${ }^{14}$

Uno de los métodos tradicionales para abordar el problema de la endogeneidad es la instrumentación de las variables que se consideran endógenas. En la literatura tradicionalmente se han utilizado como instrumentos: la edad de la primera relación sexual, la edad de la menarquia y el uso de anticonceptivos en la primera relación sexual. ${ }^{15}$

Aunque la literatura previa para el caso colombiano ha utilizado esta estrategia para medir otros resultados de la maternidad adolescente, los resultados arrojan conclusiones que, aunque son comparables con estudios anteriores, parecen incrementar los sesgos. Esto puede estar explicado por la dificultad de encontrar instrumentos certeramente exógenos o la debilidad de los mismos. Por lo tanto, en este trabajo se adopta una estrategia de identificación diferente.

\subsection{Estrategias de identificación}

Para poder obtener un estimador insesgado de la fecundidad sobre en la oferta laboral de los individuos, se plantean dos alternativas metodológicas complementarias. Primero, se controla por el sesgo de selección explicado por variables observables, a través de emparejamiento. Segundo, se busca resolver el sesgo de selección (en observables y no observables) de ser padre joven, tomando una muestra de solo padres jóvenes y evaluando el impacto de un segundo hijo a edad temprana.

\subsubsection{Primer método}

Como se mencionó anteriormente, se trata de controlar por la heterogeneidad observable, empleando técnicas de correspondencia (Propensity Score Matching). Véase (Roy, 1951;

\footnotetext{
${ }^{14}$ Inicialmente, se buscó corregir la endogeneidad haciendo uso de los abortos naturales, siguiendo a (Hotz, 2005; Acevedo et al., 2012), para comparar las jóvenes que habían experimentado este tipo de abortos con sus pares que sí dieron a luz. No se obtuvieron resultados confiables, por el bajo volumen de reportes de estos abortos espontáneos en las encuestas a hogares. Con las bases de datos disponibles en Colombia es imposible construir tal variable y por tanto seguir esta estrategia. Cabe recalcar que este tipo de análisis excluye el posible efecto de la paternidad joven en los ingresos.

${ }^{15} \mathrm{La}$ GEIH no contiene preguntas que permitan usar los instrumentos tradicionales, se buscó seguir el método de instrumentación de Klepinger (1997), pero los resultados no parecían reducir los sesgos describe la teoría.
} 
Rubin, 1974; Rosenbaum \& Rubin, 1983) ${ }^{16}$. La técnica se basa en que al no ser factible observar al padre en dos situaciones contra-factuales (ingreso laboral habiendo sido padre joven o habiendo postergado su paternidad), se contrastan los resultados de un grupo de tratamiento (padres a edad temprana ${ }^{17}$ ) con los de un grupo de control (padres que postergaron su paternidad) que se supone comparable dadas sus características observables, así pues, las diferencias entre ambos grupos se atribuirían únicamente al hecho de haber sido padre joven.

Esta técnica resume un vector de variables $x$, previas al tratamiento de cada individuo, en una única variable $e(x)$, la cual se define como la probabilidad de ser padre joven condicionada a las características previas a la paternidad. Donde $0<e(x)<1$. Finalmente, se mide el Efecto promedio del tratamiento sobre los tratados (ATT).

$$
\tau_{A T T}=\tau=E\left[E\left\{Y_{1 i} \mid D_{i}=1, e\left(x_{i}\right)\right\}-E\left\{Y_{0 i} \mid D_{i}=0, e\left(x_{i}\right)\right\} \mid D_{i}=1\right]^{18}
$$

Donde $D_{i}$ que es igual a uno si el individuo $i$ recibe el tratamiento (en nuestro caso que sea un padre joven) y cero de lo contrario. El resultado potencial estaría definido como $Y_{i}\left(D_{i}\right)$ para cada individuo $i$, donde $Y_{i}$, para este estudio, es el logaritmo del ingreso laboral por hora.

Esto se puede conseguir si se cumplen los siguientes supuestos: Primero, hay un equilibrio en el set de características individuales $D \perp x \mid e(x)$, es decir, individuos con un mismo valor $e(x)$ deben tener la misma distribución de características individuales independientemente si son o no padres jóvenes. Segundo, se cumple el supuesto de independencia condicional $(Y(0), Y(1) \perp D \mid e(x))$, lo que quiere decir que las diferencias son capturadas en $x$, y las variables resultado son independientes del estado de ser o no padre joven (Rubin, 1977). Así, la variable resultado $Y(0)$ condicionada en $x$ posee la misma función de distribución que la variable resultado $Y(0)$ que los padres jóvenes

\footnotetext{
16 Formalmente, Rosenbaum y Rubin (1983) definen el propensity score como como la función más basta de covariables resumidas en un puntaje balanceado. La puntuación balanceada es una función de covariables pre-tratamiento observadas da forma a la distribución condicional de las covariables dada la puntuación de equilibrio es la misma para tratados y controles.

${ }^{17}$ La variable padre joven o padres a edad temprana comprende tanto a madres como padres que tuvieron sus hijos antes de los 21 años.

18 Para un detalle técnico del funcionamiento del Propensity Score Matching. Véase (Bernal R. \& Peña X., 2011)
} 
hubiesen obtenido si hubieran postergado la maternidad/paternidad. Esta hipótesis exige que el investigador conozca todas las variables que influyen sobre los resultados y sobre la probabilidad de ser padre joven. Debido a que la estructura de datos hace imposible llegar a esta situación, es necesario incluir variables que permitan llegar a una aproximación razonable de esta hipótesis. Tercero, se debe cumplir el supuesto de participación unitaria estable, es decir, que el impacto que tenga la maternidad/paternidad temprana sobre una variable de resultado de un individuo no depende del estatus de paternidad de otros individuos. En otras palabras, el resultado observado (ingreso laboral) para un padre joven depende solo de sí mismo y no de si otros individuos son padres jóvenes. Lo anterior no quiere decir que la paternidad temprana de un individuo no este afectada por la paternidad temprana de otros individuos.

Al final, obtendríamos un estimador insesgado de la forma:

$$
E[Y=t, e(x)]=\beta_{0}+\sum_{i=1}^{n} \beta_{i} X_{i}+\tau T_{i}+\varepsilon_{i}
$$

Donde $X_{i}$ son variables de control y $\varepsilon_{i}$ es el termino del error. La interpretación del efecto del tratamiento $\tau$ depende de la escogencia de pesos, es decir, la forma en la que se compararan los tratados con uno o varios individuos del grupo de control. Cuando se usan varios individuos de control para ser comparados con un individuo tratado, se busca darle menos peso relativo o nulo a aquellos individuos que son menos comparables dado su valor $e(x)$. Este estudio se concentra en el emparejamiento de Kernel (Epanechnikov), el cual, se resume una función de distribución que asigna menos peso relativo a individuos controles menos comparables a medida que se alejan de un $e\left(x_{i}\right)$ especifico.

Una vez corroborado el balance de las estimaciones se realizarán 4 especificaciones para comparar los efectos en muestras distintas. Primero se comparará a los padres a edad temprana con los demás padres. Esta especificación es la de mayor interés, pues prueba la hipótesis de una extra-penalidad en el ingreso de los padres por el hecho de haberlo sido a edad temprana. Segundo, comparamos a los padres en edad temprana con padres que 
postergaron su paternidad y con los no padres. Esta estimación fortalece la hipótesis anterior, y al interpretar los resultados con los de la primera especificación permiten determinar qué proporción del impacto en los ingresos de ser padre se explica por haber sido padre joven. En la tercera especificación se compara a los padres a edad temprana con los no padres. Al comparar los resultados con los de la primera especificación nos ayuda a explorar qué tan distinto es no tener hijos o postergar la maternidad/paternidad. Por último, reducimos la muestra a aquellos padres que tienen un solo hijo y comparamos a los padres a edad temprana con el resto de padres, para determinar si el evento de ser padre joven, en sí mismo, explica la totalidad del efecto o es necesario indagar sobre la presencia de más hijos como un canal potenciador de la penalidad.

Para observar el efecto heterogéneo por sexo, se realizarán estimaciones independientes para hombres y mujeres de la primera especificación. Este procedimiento simétrico para hombres y mujeres es razonable, ya que no existen diferencias estadísticamente significativas entre las edades de las parejas de la muestra seleccionada. Así mismo, para aislar el posible impacto del embarazo adolescente, se evaluarán las cuatro especificaciones en una muestra que omite a los padres adolescentes y toma como grupo de tratamiento a aquellos padres que lo fueron entre los 18 y 21 años.

\subsubsection{Segundo método}

En el primer método se corrige el sesgo de selección en variables observables. En esta segunda estrategia de identificación nos concentramos en una muestra de padres jóvenes entre 25 y 35 años que sean padres de 2 hijos $^{19}$. En este ejercicio compararemos el impacto sobre los ingresos de tener un segundo hijo a edad temprana con haberlo postergado a una edad más avanzada, condicional en haber tenido el primer hijo a edad temprana. Al enfocarnos en este grupo se resuelve el sesgo de selección (en observables y no observables) de ser padre joven. Este análisis nos permite explorar el impacto de tener un segundo hijo sobre los ingresos, si este fue concebido también siendo padre joven. Si bien la concepción del segundo hijo a edad temprana no es del todo aleatoria, es presumiblemente menos endógena pues la decisión de tener otro hijo podría estar más

\footnotetext{
${ }^{19}$ El 77\% de los padres entre 25 y 35 años han sido padres de 1 o 2 hijos, por lo que para esta estrategia contamos con una muestra de 18.460 observaciones.
} 
ligada a las preferencias de los individuos, ya que la experiencia de haber tenido un hijo a esta edad les permite evaluar de mejor manera las conveniencias del momento en el que desean su segundo hijo. Siguiendo a Olarte y Peña (2010), se evaluará una ecuación de Mincer del tipo:

$$
\log (Y / h r)=\beta_{0}+\beta_{1} X i+\beta_{2} X h+\beta_{3} X l+\beta_{4} D_{i}+\varepsilon
$$

Donde $X i$ es un vector de características individuales y de capital humano (sexo, edad, edad al cuadrado, nivel educativo, jefatura de hogar, estado civil), $X h$ es un vector de características del hogar (estrato socio económico y ruralidad) , $X l$ es un vector de características geográficas (región) ${ }^{20}, D_{i}$ es un indicador que toma valor igual a 1 si el segundo hijo también fue concebido de manera temprana y cero de lo contario, es decir, si el segundo hijo se postergo a edades más avanzadas. Se realizan estimaciones independientes para hombres y mujeres. Como esta muestra solo contemplaría información de padres ocupados, se corrige el sesgo de autoselección a través del método de Heckman (1979). Para hacer la estimación de la probabilidad de trabajar se sigue a Olarte y Peña (2010), incluyendo como variables de predicción: los ingresos no laborales del individuo, los ingresos de los otros miembros de hogar, y si uno de los miembros del hogar tiene título de propiedad sobre la vivienda. No resulta necesario buscar métodos de predicción independientes para hombres y mujeres puesto que el sesgo de selección está concentrado en las mujeres. ${ }^{21}$

En conjunto, las dos estrategias se complementan, pues la segunda estrategia confirma la existencia de la penalidad, aun cuando se tienen en cuenta características no observables. Lo que respalda los resultados del primer método. Además establece que la presencia de un hijo adicional siendo joven potencia los resultados negativos.

\subsection{Emparejamiento: Predicción de la maternidad temprana}

Para la construcción del modelo de predicción de la paternidad o maternidad temprana $e(x)$ de la ecuación (2), se empleó un stepwise regression, seguido de Klepinger et al. (1997)

\footnotetext{
${ }^{20}$ Las estadísticas descriptivas de las variables tomadas para este modelo y la corrección de Heckman se encuentran en el apéndice 1, cuadro 1.2

${ }_{21}$ El estudio de Olarte y Peña (2010) es para mujeres. La tasa de ocupación de los hombres para la muestra seleccionada es de $86,2 \%$ por lo que el sesgo de selección no constituye un problema fundamental para estos.
} 
para encontrar el modelo que mejor se ajustara a la predicción a través de un modelo Probit. Así pues, se involucraron mediciones de características geográficas (variables dicótomas de ciudad y ruralidad), individuales (edad, sexo, parentesco) y a nivel de hogar (educación del jefe de hogar ${ }^{22}$, estrato socio económico: bajo, medio y alto) de los padres. Además, se incorporó la tasa de fecundidad temprana por municipio ${ }^{23}$ a nivel agregado anual que es una variable contextual (proveniente de registros administrativos ${ }^{24}$ ) que teóricamente se consideraran predictores plausibles de la paternidad temprana; se vinculó a la base a través de la variable municipio ${ }^{25}$. Para poder usarse esta variable, fue necesario controlar por el hecho de que la población no fuera migrante. ${ }^{26}$

Así pues, habiendo introducido potenciales covariables de comunidad y contexto, se procedió a buscar un elemento que afectara las decisiones individuales, para lo cual, se utilizó un algoritmo en $\mathrm{sql}^{27}$ que construyera una variable que identificara el número de personas que residían en el hogar del individuo, los cuales eran mayores y a su vez también habían sido padres jóvenes. Desde el punto de vista teórico, East (1999) demuestra que si en el hogar existen historias de maternidad temprana, se generan cambios en las estructuras familiares, soporte familiar y asistencia al cuidado del niño, que crean un contexto en el que hermanos menores pueden aumentar la probabilidad de ser padres jóvenes, asumiendo comportamientos más riesgosos. El autor, respaldado por que más del $80 \%$ de las adolescentes continúan en su hogar al menos un año después de ser madres observa

\footnotetext{
${ }^{22}$ Condicionado a que el padre joven no sea el jefe del hogar.

23 La tasa de fecundidad temprana del municipio, resultó ser una variable apropiada y significativa. Intuitivamente esta variable podría ser respaldada por Flórez y Núñez (2002), quienes sugieren que la maternidad adolescente está influenciada por las condiciones idiosincráticas locales. Para los autores, las jóvenes que residen en departamentos con altas tasas de fecundidad están en mayor riesgo de ser madres adolescentes: existen factores culturales que las presionan a tener los hijos a edad temprana. Este trabajo extiende la afirmación de los antes mencionados, aseverando que el contexto social en el que están inmersos los jóvenes, los conduce a concebir la paternidad temprana como una regla natural dentro del ciclo de vida, en otras palabras, los pares de los jóvenes se convierten en un elemento fundamental para la toma de este tipo de decisiones. Esta variable se detalla a nivel municipal, sorpresivamente las tasas de fecundidad temprana son bastante heterogéneas entre municipios, aun del mismo departamento, y además poco volátiles a través de los años. En este sentido, se asignó a cada padre la tasa promedio de fecundidad temprana del municipio (donde ha residido siempre).

${ }^{24}$ Cifras del registro de nacido vivo de estadísticas vitales EV-DANE y el registro civil de nacimiento de la Registraduria Nacional. 25 Después de utilizar la metodología de Klepinger et al. (1997) antes señalada, se descartaron del modelo: el gasto público departamental en atención al embarazo adolescente y un índice económico a nivel de hogar, como lo formula Madrigal (1986), donde se tiene en cuenta una ponderación sobre la tenencia de electrodomésticos, acceso a servicios públicos y algunos bienes a través de la metodología de componentes principales.

26 Según datos del tercer seguimiento de la encuesta longitudinal para la evaluación del programa Familias en Acción. Datos calculados para un total de 4579 madres entrevistadas. Se muestra que, aproximadamente el $70 \%$ de las madres que lo fueron a la edad de 20 o 21 años deseaban serlo en ese momento y no consideraban la opción de postergarlo. Para las madres que lo fueron a los 18 años, $63,9 \%$ querían quedar embarazadas a esa edad. Esto apoya la idea de la influencia existente de los pares en la toma de decisiones frente a la edad de concebir el primogénito. Y aunque se trata de una población específica (población beneficiaria o elegida del programa Familias en Acción), esta es una de las poblaciones donde mayor incidencia de embarazo a edad temprana (cerca del $76 \%$ de las madres entrevistadas fueron antes de los 21 años). Cabe resaltar que el deseo por los hijos no es una variable que desde el punto de vista demográfico se considere certera, por razones culturales y sociológicas.

${ }^{27}$ Structured query language es un lenguaje declarativo de acceso a bases de datos relacionales que permite especificar diversos tipos de operaciones en ellas.
} 
impactos intergeneracionales que trae para el hogar una historia de maternidad temprana, encuentra que en los hogares donde hay una madre o padre adolescente, decrece el monitoreo y se incrementa la aceptabilidad de la sexualidad adolescente.

Otras líneas de investigación desde la psicología y la demografía apoyan este resultado, (Meade et al. 2008; Joan y Kay 1992), las hijas de madres adolescentes son promedio 66\% más propensas a convertirse en madres adolescentes y así perpetuando un ciclo intergeneracional. Explicando que no se debe a factores biológicos como la edad de la pubertad sino a las condiciones socioeconómicas y contextos familiares donde los jóvenes crecen $^{28}$.

En este orden, a cada individuo de la muestra seleccionada se le construyeron tres variables dicótomas: presencia de una persona mayor que ha sido padre joven, presencia de dos personas mayores que han sido padres jóvenes, presencia de tres o más personas que han sido padres jóvenes.

\subsection{Canales}

Este trabajo desarrolla dos hipótesis sobre el conducto a través del cual los padres jóvenes experimentan una penalidad en sus ingresos. La primera de ellas determina que la maternidad/paternidad joven se presenta en un momento crítico de la vida, donde aumenta la probabilidad de interrumpir eventos importantes en la formación de capital humano. Este factor es fuerte para entender la penalidad pero puede no explicar la totalidad del impacto. Por esto la segunda hipótesis plantea que el abandono escolar puede estar sucedido por cambios en la forma de la vinculación al mercado laboral. Es posible que los padres jóvenes tengan ingresos más bajos porque su salario de reserva desciende al volverse más impacientes para ingresar al mercado laboral. Necesitando emprender una actividad generadora de ingresos para el mantenimiento económico del niño, pueden ingresar a realizar actividades que no hubiesen aceptado si no fuesen padres o hubiesen esperado más tiempo para ingresar. De esta manera, su primera incursión al mercado de trabajo puede no darse en condiciones óptimas. Una primera vinculación al mercado de trabajo en condiciones deterioradas, podría explicar en gran medida los ingresos futuros de los

\footnotetext{
${ }^{28}$ Ambos estudios, Meade (2008) y Jon y Kay (1992) fueron investigaciones realizadas con aproximaciones distintas para Estados Unidos.
} 
individuos, al menos en el mediano plazo (Oreopoulos et al., 2006; Oyer, 2006; Kahn, 2010; Brunner y Kuhn, 2010).

Haciendo uso del Propensity Score, en concreto, la especificación (i), se evaluarán diferentes resultados asociados y determinantes tradicionales del ingreso laboral de los individuos en dos vías: La primera, una evaluación sobre los choques a la acumulación de capital humano de los padres jóvenes, y la segunda, un análisis sobre el salario de reserva y la vinculación al mercado de trabajo.

Uno de los principales inconvenientes para determinar si la maternidad genera ausentismo escolar, es la simultaneidad de la decisión educarse o ser padre. En la literatura previa sobre el tema se suele convivir con la encrucijada de si la madre abandonaba sus estudios por causas de maternidad temprana, o si el abandono escolar era la causa de la maternidad temprana. Este estudio resuelve ese sesgo, gracias a que los datos de la GEIH indagan si los padres abandonaron permanente o temporalmente sus estudios, o su trabajo, por razones de maternidad o paternidad (ver apéndice 4). Estas variables se convertirán en la principal herramienta de análisis para observar el efecto en la acumulación de capital humano, junto con un análisis sobre la probabilidad de alcanzar títulos educativos.

Para evaluar el efecto de la vinculación al mercado de trabajo (ya que el salario de reserva no es observable) se utilizará como aproximación el salario del primer empleo y una caracterización la primera incursión de los padres al mercado de trabajo, para esto, se tomará una muestra de padres ocupados que no hayan tenido un trabajo distinto al que tienen actualmente, esto permitirá identificar el momento en que se vincularon al trabajo y bajo qué condiciones lo hicieron (horas trabajadas, trabajo de medio tiempo por causa de responsabilidades familiares, continuidad laboral, ocupación, indicadores de informalidad y subempleo por horas y competencias ${ }^{29}$ ). Del mismo modo se estudiaran las variables sobre abandono temporal o permanente de trabajo por razones de maternidad/paternidad, para examinar las posibles consecuencias de la maternidad/paternidad temprana sobre la

\footnotetext{
${ }^{29}$ La informalidad se mide desde dos definiciones: La primera, es la definición DANE, que son los ocupados en empresas de hasta 10 trabajadores, exceptuando a los profesionales que trabajan en su oficio. La segunda, es la aproximación tradicional sobre si el ocupado hace cotización al sistema de seguridad social en salud y pensión.

El subempleo por horas y competencias se refiere a la manifestación de inconformidad con el número de horas trabajadas, deseando y pudiendo trabajar más horas, o porque su labor no está acorde a sus competencias personales y está buscando trabajo.
} 
experiencia laboral, además se analizará la probabilidad de rechazar ofertas laborales por causa de maternidad/paternidad.

Por último se agregará una estimación de la probabilidad de tener más hijos en el mediano plazo, para evaluar qué tanta relevancia tienen los resultados del segundo modelo.

Estudios previos (Flórez y Núñez 2002) sugieren que los efectos de la paternidad adolescente en el abandono escolar pueden ser menos importantes para los hombres, razón por la cual los canales de transmisión del efecto sobre los ingresos pueden resultar heterogéneos para hombres y mujeres, por tanto, se realizan estimaciones del ATT por separado.

\section{Resultados}

\subsection{Emparejamiento}

Las estimaciones basadas en la GEIH confirman que los individuos que, en el momento de la entrevista tenían entre 25 y 35 años y que se convirtieron en padres a edad temprana (PETem), presentan una penalidad significativa en los ingresos laborales frente a los padres que postergaron su paternidad. La construcción del propensity score $e(x)$ arrojó un área importante de soporte común, que permitió un satisfactorio emparejamiento entre individuos homogéneos dadas sus características observables.

\section{Gráfica 3. Densidad del propensity score estimado $e(x)$}




Los resultados son robustos frente a cualquier método de emparejamiento escogido (ver apéndice 2, cuadro 2.1). Nos concentraremos en los resultados del emparejamiento de Kernel (Epanechnikov) ya que los coeficientes estimados bajo los métodos de distancia máxima y Kernel resultaron, en general, siendo balanceados entre las muestras de tratados y controles. La gráfica 4 ilustra el porcentaje de sesgo o diferencias de las covariables entre tratados - controles antes y después del emparejamiento, entre más alejado este el indicador de la línea vertical señala una mayor diferencia entre los dos grupos, después del emparejamiento las muestras se encuentran balanceadas, es decir, no existen diferencias estadísticamente significativas entre tratados y controles, al menos en las características observables.

Habiendo validado el balance de la muestra, el cuadro 4. presenta los resultados del ATT para cuatro especificaciones en una muestra de individuos no migrantes entre 25 y 35 años. (i) Padres a edad temprana en el total de padres, (ii) Padres a edad temprana comparados con padres y no padres, (iii) Padres a edad temprana frente a no padres, (iv) Padres a edad temprana en una muestra de padres con un solo hijo. 
Gráfica 4. Test grafico de balance del método Kernel (Epanechnikov), diferencias entre tratados y controles antes y después del emparejamiento.

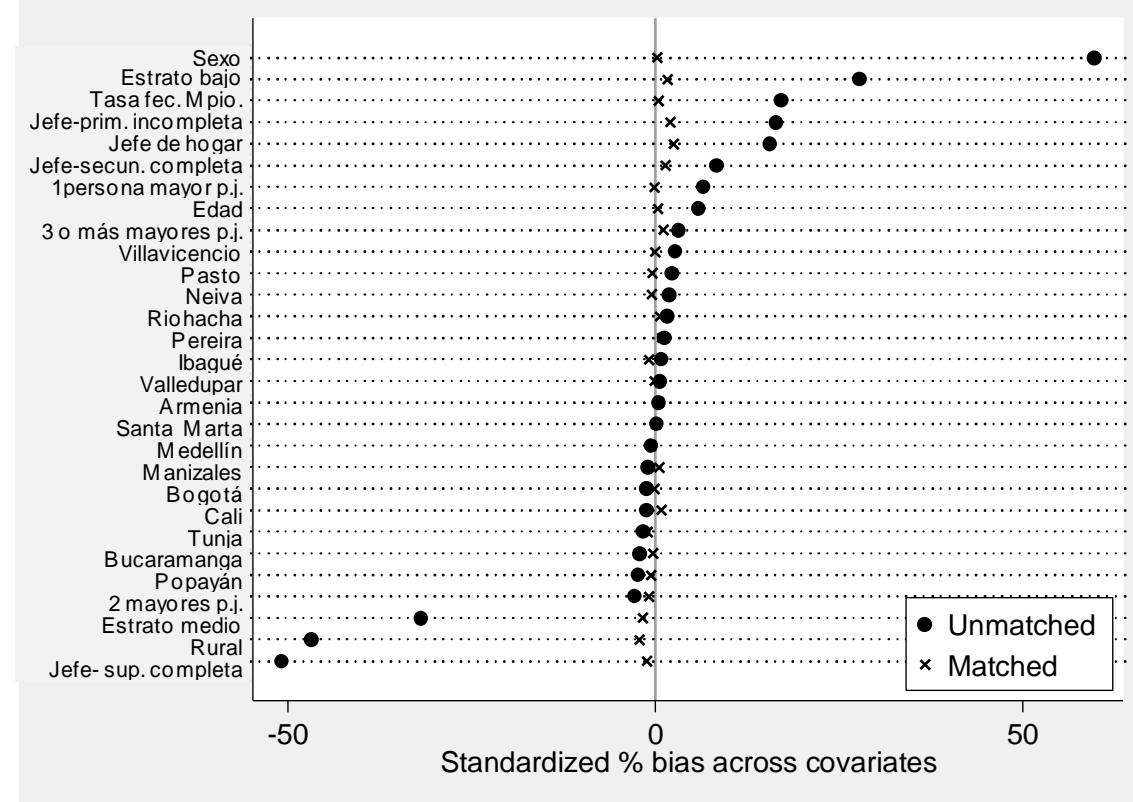

La especificación (i) concentra nuestro principal interés, la comparación de los ingresos laborales por hora en una muestra de padres. Bajo el método de Kernel se encuentra que, en efecto, los padres a edad temprana presentan una penalidad en los ingresos, tienen en promedio $11,7 \%$ menos ingreso laboral por hora que sus pares que postergaron la maternidad/paternidad. Esto sugiere que existe una penalidad en los ingresos adicional a la de ser padre, que se explica por el hecho de que la concepción se dé a edades tempranas.

La especificación (ii) complementa el análisis anterior, nos muestra que al comparar los ingresos de los padres a edad temprana frente a los padres que postergaron su paternidad, y además los que no fueron padres, la situación negativa se acentúa pero no en gran medida, los padres jóvenes reflejan un impacto negativo de los ingresos de 12,5\%. Este resultado, contrastado con el resultado de la especificación (i), sugiere que gran parte del impacto en los ingresos de ser padre se explica por haber sido padre joven, nos propone que los estudios previos que se realizaron para mujeres y que valoraban el impacto del hecho de ser madre sobre los ingresos, estarían explicados en buena medida por el hecho de haber sido madre a edad temprana y no simplemente por ser madre. 
Cuadro 4. Efecto de la maternidad o paternidad temprana sobre los ingresos laborales. Resultados de las estimaciones por el método de Propensity Score Matching hacen referencia al ATT, para una muestra de no migrantes entre 25 y 35 años.

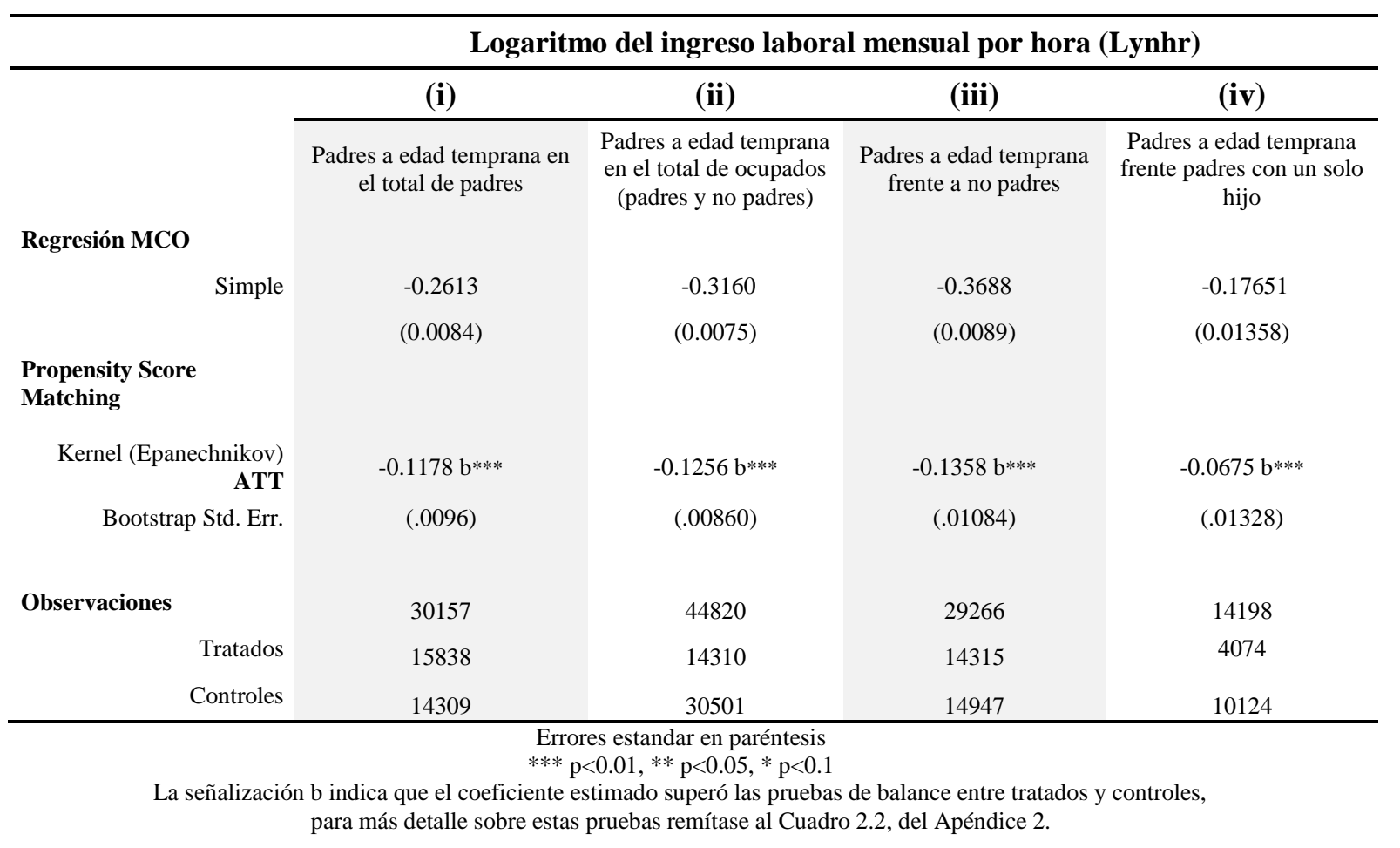

La especificación (iii) compara padres a edad temprana frente a no padres, omitiendo de la muestra a los individuos que postergaron su paternidad, aquí se observa un coeficiente negativo de 13,5\%, comparable al calculado para las mujeres en previos estudios (Olarte $\&$ Peña, 2010. [17,6\%]). Esto, soportaría la hipótesis de que los resultados de postergar la paternidad/maternidad estarían más cerca de los resultados de no haber sido padre.

Es necesario resaltar, que cuando en el grupo de controles se contemplan los no padres, podemos incluir sesgos resultantes de que al no poder controlar variables inobservables esta comparación puede ser ruidosa. La decisión de no ser padres puede estar correlacionada con las habilidades en el mercado de trabajo. Aun así, más que ofrecer conclusiones, respaldan el análisis del impacto resultante de la estimación (i).

La estimación (iv) toma una muestra de padres de un solo hijo por dos razones. La primera, es seleccionar una población de cierta manera más homogénea, corrigiendo el posible sesgo del número de hijos. La segunda, es observar si el evento de ser padre joven en sí mismo (solo tener un hijo a esa edad) genera distorsiones capaces de explicar los efectos 
encontrados en las especificaciones anteriores, o si por el contrario existen otras variables como el número de hijos que valen la pena estudiar.

Así pues, para esta especificación se encontró que los padres de un solo hijo que lo tuvieron a edad temprana ganan en promedio 6,7\% menos que los padres que tuvieron un único hijo después de los 21 años. Este resultado plantea que, aunque ser padre de un hijo a edad temprana explica casi la mitad del impacto medido en la especificación (i), es necesario analizar la presencia de más hijos y el momento en que estos se conciben como un factible potenciador del impacto sobre los retornos laborales, asunto que se tratará en la siguiente estrategia y en la sección de los canales.

El cuadro 5. muestra los resultados de la especificación (i) (nuestro principal interés) desagregada por sexo, en efecto, ser padre joven no solo es más preponderante para las mujeres sino que el impacto en los ingresos resulta superior al que enfrentan los hombres, aunque para ellos, el impacto existe y es significativo.

Para las mujeres madres, el hecho de concebir su primer hijo a edad temprana impacta negativamente sus ingresos por hora en un 12,6\%, para los hombres la penalidad en los ingresos es del orden del $5,1 \%$, frente a los padres que postergaron su paternidad ${ }^{30}$. Además de confirmar que existe una penalidad para los hombres, este resultado es interesante desde un enfoque de género. En primera instancia, la diferencia del impacto nos impulsa a explorar los canales de transmisión diferenciados por sexo, los efectos pueden ser heterogéneos a causa de que biológicamente un embarazo está a cargo de la mujer y las interrupciones en ciclos de formación de capital humano son inminentes, mientras que para los hombres no lo es así, es probable que este factor no sea explicativo de la penalidad para estos últimos. El impacto sustancialmente menor en los hombres puede estar explicado por qué algunos no asumen la responsabilidad de ser padre y dejan en manos de la mujer el cuidado y el mantenimiento económico del niño. Por otra parte, destaca la posibilidad de que la maternidad temprana sea un factor explicativo adicional sobre las brechas salariales entre hombres y mujeres.

\footnotetext{
${ }^{30}$ Para las dos estimaciones se obtuvieron coeficientes que cumplieron los test de balance entre tratados y controles. Ver cuadro 2.4 del apéndice 2 .
} 
Cuadro 5. Efecto (ATT) de la maternidad o paternidad temprana sobre los ingresos laborales. Resultados de las estimaciones del efecto de la maternidad o paternidad según sexo.

\begin{tabular}{|c|c|c|}
\hline & \multicolumn{2}{|c|}{$\begin{array}{c}\text { Logaritmo del ingreso laboral } \\
\text { mensual por hora (Lynhr) }\end{array}$} \\
\hline & \multicolumn{2}{|c|}{ Padres a edad temprana en el total de padres (i) } \\
\hline & Hombres & Mujeres \\
\hline \multicolumn{3}{|l|}{ Propensity Score Matching } \\
\hline Kernel (Epanechnikov) ATT & $-0.0510 b^{* * *}$ & $-0.1263 b^{* * *}$ \\
\hline Bootstrap Std. Err. & $(0.00989)$ & \\
\hline Observaciones & 16990 & 16519 \\
\hline Tratados & 6083 & 6790 \\
\hline Controles & 10907 & 6729 \\
\hline \multicolumn{3}{|c|}{$\begin{array}{l}\text { Errores estándar en paréntesis } \\
* * * p<0.01, * * p<0.05, * p<0.1\end{array}$} \\
\hline
\end{tabular}

Dado que gran parte la literatura que guarda relación con este trabajo está concentrada en el análisis del embarazo adolescente, y que se han demostrado ampliamente sus consecuencias negativas en el bienestar de los individuos, la política pública ha sido, en general, focalizada a atender este fenómeno. Por tanto, uno de los objetivos principales de este estudio es demostrar que existen implicaciones negativas de ser padre joven, independientemente de ser padre adolescente. Para este fin, se realizó una estimación que omitía de la muestra a todos aquellos individuos que fueron padres adolescentes y se evaluaron de nuevo las cuatro especificaciones. Los resultados del cuadro 6 muestran los coeficientes obtenidos después de omitir a los padres adolescentes, estos resultan casi inalterados con respecto de los resultados del cuadro 4. Se podría llegar a argumentar que, un poco más del $80 \%$ del impacto observado en los ingresos, esta explicado por ser padre joven y no por ser padre adolescente. 
Cuadro 6. Efecto de la maternidad o paternidad temprana sobre los ingresos laborales. Resultados de las estimaciones del efecto de la maternidad o paternidad entre los 18 y 21 años.

\begin{tabular}{|c|c|c|c|c|}
\hline & \multicolumn{4}{|c|}{ Logaritmo del ingreso laboral mensual por hora (Lynhr) } \\
\hline & (i) & (ii) & (iii) & (iv) \\
\hline & $\begin{array}{c}\text { Padres a edad } \\
\text { temprana en el total } \\
\text { de padres }\end{array}$ & $\begin{array}{l}\text { Padres a edad temprana } \\
\text { en el total de ocupados } \\
\text { (padres y no padres) }\end{array}$ & $\begin{array}{l}\text { Padres a edad temprana } \\
\text { frente a no padres }\end{array}$ & $\begin{array}{c}\text { Padres a edad temprana } \\
\text { frente padres con un solo } \\
\text { hijo }\end{array}$ \\
\hline \multicolumn{5}{|l|}{ Propensity Score Matching } \\
\hline Kernel (Epanechnikov) ATT & $-0,0913 b^{* * *}$ & $-0,0998 b^{* * *}$ & $-0,1059 b^{* * *}$ & $-0,0668 b^{* * *}$ \\
\hline Bootstrap Std. Err. & $(.00866)$ & $(.00757)$ & $(.01104)$ & $(.01472)$ \\
\hline Observaciones & 26010 & 40673 & 25119 & 13457 \\
\hline Tratados & 10169 & 10168 & 10170 & 3328 \\
\hline Controles & 15838 & 30501 & 14947 & 10124 \\
\hline La señalizació & $\begin{array}{r}\text { Err } \\
* * * \\
\text { Errores calculados } \\
\text { ca que el coeficiente } \\
\text { nás detalle sobre est }\end{array}$ & $\begin{array}{l}\text { res estándar en paréntesis } \\
<0.01, * * \mathrm{p}<0.05, * \mathrm{p}<0 . \\
\text { on el método Bootstrap co } \\
\text { stimado superó las pruebr } \\
\text { pruebas remítase al Cuac }\end{array}$ & $\begin{array}{l}60 \text { iteraciones } \\
\text { de balance entre tratados } \\
2.5 \text { del Apéndice } 2 .\end{array}$ & controles, \\
\hline
\end{tabular}

Esto fortalece la idea de que la baja edad media de procreación inicial de los colombianos resulta inconveniente en términos de los retornos laborales. Dicho de otra forma, la edad promedio en la que los colombianos tienen sus hijos, al estar cercana al rango entre los 18 y 21 años, está generando efectos nocivos sobre los ingresos de los jóvenes en el mediano plazo.

\subsection{Impacto de un hijo adicional siendo joven}

El cuadro 7 muestra los resultados obtenidos de explorar el impacto de un hijo adicional mientras se es joven. Esta estimación toma una muestra de padres de dos hijos que tuvieron su primer hijo a edad temprana. Se realiza la corrección del sesgo de selección bajo el método de Heckman (1979). Se demostró que la corrección es estadísticamente significativa, por lo que los coeficientes estimados son consistentes, razón por la cual se interpretarán estos y no los obtenidos por M.C.O.

Se encontró que aquellos padres que concibieron su segundo hijo también siendo jóvenes registran un ingreso por hora 5,7\% inferior a aquellos padres jóvenes que decidieron 
postergar su segundo hijo. En la misma vía que el modelo anterior, las mujeres reciben la mayor parte de este impacto $(8,6 \%)$, para los hombres la penalidad es de $3,2 \%$.

Esta forma de evaluación corrige el sesgo de endogeneidad de ser padre joven, atribuido a características no observables, debido a que toma una muestra de solo padres jóvenes, y aunque se es consciente de que un segundo hijo a edad temprana no es del todo aleatorio, supone que la decisión del momento en que concebirán un segundo hijo, dependerá de las preferencias que tengan los individuos sobre su segunda paternidad, dada la experiencia previa de haber concebido un hijo en esta etapa.

Estos resultados nos ofrecen tres conclusiones importantes: primero, aun corrigiendo por la endogeneidad que genera ser padre joven, confirma que el hecho de tener hijos en esta etapa de la vida genera impactos negativos en los ingresos. Segundo, el número de hijos concebidos a edad temprana tiene repercusiones sobre los ingresos de los padres jóvenes y puede ser un eslabón dentro de los canales por los cuales se transmite este impacto.

Cuadro 7. Efecto de un segundo hijo a edad temprana sobre los ingresos laborales. Resultados de las estimaciones por M.C.O y Máxima verosimilitud (Heckit) para una muestra de padres jóvenes con dos hijos. ${ }^{31}$

\begin{tabular}{|c|c|c|c|c|c|c|}
\hline \multicolumn{7}{|c|}{ Logaritmo del ingreso laboral mensual por hora } \\
\hline & \multicolumn{2}{|c|}{ Total } & \multicolumn{2}{|c|}{ Mujeres } & \multicolumn{2}{|c|}{ Hombres } \\
\hline VARIABLES & $(\mathrm{MCO})$ & (Heckit) & $(\mathrm{MCO})$ & (Heckit) & $(\mathrm{MCO})$ & (Heckit) \\
\hline \multirow[t]{2}{*}{$\begin{array}{l}\text { Segundo hijo concebido } \\
\text { siendo joven }\end{array}$} & $-0.044 * * *$ & $-0.057 * * *$ & $-0.038^{* * *}$ & $-0.086^{* * *}$ & $-0.045^{* * *}$ & $-0.032 * * *$ \\
\hline & $(0.002)$ & $(0.0021)$ & $(0.0027)$ & $(0.0032)$ & $(0.0027)$ & $(0.0028)$ \\
\hline \multicolumn{7}{|l|}{ CONTROLES } \\
\hline Individuales & $\sqrt{ }$ & $\sqrt{ }$ & $\sqrt{ }$ & $\sqrt{ }$ & $\sqrt{ }$ & $\sqrt{ }$ \\
\hline Hogar & $\sqrt{ }$ & $\sqrt{ }$ & $\sqrt{ }$ & $\sqrt{ }$ & $\sqrt{ }$ & $\sqrt{ }$ \\
\hline Geográficos & $\sqrt{ }$ & $\sqrt{ }$ & $\sqrt{ }$ & $\sqrt{ }$ & $\sqrt{ }$ & $\sqrt{ }$ \\
\hline Observaciones & 11,522 & 17,353 & 7,243 & 12,644 & 4,279 & 4,709 \\
\hline R-squared & 0.187 & & 0.180 & & 0.172 & \\
\hline Test Wald & & $* * *$ & & $* * *$ & & $* *$ \\
\hline
\end{tabular}

\footnotetext{
${ }^{31}$ Las estadísticas descriptivas de las variables incluidas en esta estimación se encuentran en el Apéndice 1. Cuadro 1.2
} 
Tercero, aun después de incluir controles de capital humano, estos no son suficientes para explicar el diferencial de ingresos, lo que nos conduce a pensar que las interrupciones o el abandono escolar no explican la totalidad de la penalidad salarial.

\subsection{Canales}

Uno de los aportes más importantes del presente trabajo es la identificación del abandono de los estudios, el abandono del trabajo y el rechazo de oportunidades laborales, por razones de maternidad o paternidad. Esto es posible gracias a variables con las que antes no se contaba en las bases de datos sobre estos temas, esta información indaga de forma directa si el abandono fue explicado certeramente por la maternidad o paternidad.

En el cuadro 8.1, se exploran las que se suponen son las primeras implicaciones por las cuales se generarían impactos en los ingresos de los padres jóvenes, es decir, las atribuidas a la acumulación de capital humano.

Cuadro 8.1 Impactos a la formación de capital humano de paternidad/maternidad temprana. Resultados de las estimaciones hacen referencia al ATT, para una muestra de padres ocupados no migrantes entre 25 y 35 años.

\begin{tabular}{|c|c|c|c|c|c|c|}
\hline \multirow[b]{4}{*}{ Abandono estudios permanente } & & & & & & \\
\hline & \multicolumn{6}{|c|}{ ATT } \\
\hline & \multicolumn{2}{|c|}{ TOTAL } & \multicolumn{2}{|c|}{ Mujeres } & \multicolumn{2}{|c|}{ Hombres } \\
\hline & $.05184 * * *$ & $(.00190)$ & $.05783 * * *$ & $(.00562)$ & $.04198 * *$ & $(.00294)$ \\
\hline Abandono de estudios & $.10197 * * *$ & $(.0012)$ & $.13152 * * *$ & $(.0051)$ & $.05852 * * *$ & $(.00236)$ \\
\hline Abandono de trabajo & $-.00770 * * *$ & $(.00179)$ & -.00919 & $(.00635)$ & .00029 & $(.00154)$ \\
\hline Bachiller & $-.02836 * * *$ & $(.00148)$ & $-.04092 * * *$ & $(.0082)$ & $-.02566 * * *$ & $(.00240)$ \\
\hline Superior & $-0.123 * * *$ & $(.00428)$ & $-0.132 * * *$ & $(.00418)$ & $-0.077 * * *$ & $(.00255)$ \\
\hline Antes tenía otro trabajo & $-.02018 * *$ & $(.00856)$ & $-.02061 * * *$ & $(.00736)$ & -.00541 & $(.00588)$ \\
\hline
\end{tabular}

Errores estándar en paréntesis; Resultados estimados a través de emparejamiento Kernel (Epanechnikov) con ancho de banda 0.05. Variables dependientes son dicótomas.

Los padres que concibieron a sus hijos a edad temprana tienen una probabilidad de 10 puntos porcentuales (p.p.) más elevada de abandonar sus estudios por razones de maternidad/paternidad en comparación con sus pares que postergan la paternidad. Este diferencial es más acentuado para las mujeres (13 p.p.) que para los hombres (6 p.p.). El hecho de que la concepción biológica de los hijos este a cargo de las mujeres tiene una incidencia potencial en este resultado, las mujeres que cursan algún ciclo de educación, deben abandonar sus estudios, al menos hasta que su integridad física y los primeros 
cuidados post-natales lo permitan. La brecha en el abandono de estudios por sexo, puede también estar explicada por la no respuesta económica del padre frente al hijo, algunos padres ceden por completo el mantenimiento económico del niño a manos de la madre y continúan con sus estudios. Las interrupciones temporales de los estudios que deben asumir las mujeres, podrían también explicar el impacto en los ingresos, el retorno a las actividades académicas podría caracterizarse por ser un regreso a una educación de menor calidad, o tener menos tiempo disponible para invertir en estas actividades, la disponibilidad de información no nos permite evaluar esta hipótesis.

Ahora bien, indistintamente del sexo, la probabilidad de abandonar los estudios de manera permanente se eleva en cerca de 5 p.p. Este es el efecto más relevante en el largo plazo. Para ambos casos, hombres y mujeres, el abandono permanente estaría asociado a la responsabilidad económica que exige el cuidado del niño. Este resultado constituiría la primera fuente por la cual la penalidad en los padres jóvenes existe, no solo porque la acumulación de capital humano se ve afectada por el abandono de un ciclo académico, sino por no que no se ingresa a un ciclo más avanzado. Una lectura alternativa de los resultados indica, es que existe una salida temporal de las mujeres de los estudios por motivos biológicos pero algunas de ellas regresan. Esas salidas del mercado de trabajo también pueden estar asociadas a las brechas en los retornos entre hombres y mujeres.

Complementando lo anterior, la probabilidad de alcanzar un título de educación superior, bien sea técnico, tecnológico o universitario, se reduce de manera sustancial para hombres (7 p.p.) y mujeres (13 p.p.) cuando se es padre joven. Así pues, la acumulación de capital humano se convierte en el primer elemento explicativo de la penalidad de los ingresos. Pero parece no ser el único al cual se le debe atribuir la totalidad del impacto, la regresión auxiliar del cuadro 3.2 del apéndice 3, muestra que individuos con el mismo nivel educativo también enfrentan diferencias salariales si tienen una historia de paternidad temprana. Esta estimación es diciente, pero debe examinarse con cuidado por el bajo volumen de muestra.

Ahora analizaremos la hipótesis del descenso del salario de reserva de los padres jóvenes. Para ello tomamos como aproximación la primera vinculación de los padres jóvenes al mercado de trabajo. Analizando una muestra de padres ocupados en su primer empleo se 
encontró que el mismo impacto antes calculado es casi exacto al que se encuentra para esta submuestra. Esto respalda la idea de que la primera inserción es la que pronostica el ingreso en el mediano plazo.

Uno de los hallazgos más importantes es que los hombres padres jóvenes ingresan antes al mercado laboral, esto puede estar relacionado directamente con el abandono de los estudios. La impaciencia causada por el afán de emprender una actividad generadora de ingresos, conduce a estos interrumpir la formación de capital humano y vincularse de manera precipitada al mercado laboral, y más aún, hacer descender su salario de reserva (aceptar trabajos que no hubieran aceptado si no hubiesen sido padres).

Los resultados muestran que los padres jóvenes son 5 p.p. más propensos a vincularse a actividades como independientes, esto se explica porque involucra un menor costo que vincularse como asalariado, insertarse al mercado de trabajo como cuenta propia, puede llegar a ser más sencillo que emprender la búsqueda de un empleo asalariado (en un mercado de trabajo con una elevada tasa de desempleo para la población joven). Cabe recordar que la posición ocupacional juega un rol importante en la explicación de los ingresos, dependiendo del nivel educativo alcanzado, cuando la vinculación no está asociada a un nivel educativo superior, los ingresos percibidos por estas actividades son sustancialmente menores a los que recibiría en un trabajo de tiempo completo como asalariado, además de no incorporar a su estructura de ingreso prestaciones sociales (primas, cesantías, aportes a seguridad social).

De igual forma, estas primeras incursiones como independiente dan forma al tipo de experiencia adquirida en la primera etapa del mercado laboral, por lo que serían más propensos a perpetuarse en estas actividades, y esto daría cuenta sobre su ingreso en el mediano plazo (Hamilton B. 2000).

Por otra parte, se encontró que las jornadas laborales para los hombres no son distintas en comparación a los padres que postergaron la paternidad, pero si están más asociadas a actividades en el sector informal donde no se cotiza a salud o pensión. Una vez insertados en un mercado laboral, donde en promedio un joven tarda 22 semanas en la búsqueda de empleo en caso de quedar desocupado, sumado a la necesidad de generar ingreso, hace 
difícil la decisión de emprender nuevas búsquedas, generando una especie de ancla a la primera vinculación.

Pasando a las mujeres, en contraste a los hombres, las madres a edad temprana experimentan una penalidad sobre la experiencia laboral, al compararse con las madres que postergaron la maternidad, estas entran después al mercado laboral. A diferencia de los hombres el abandono de los estudios no estaría caracterizado por la necesidad de ingresar al mercado laboral sino que parece estar más relacionado con el cuidado del hijo, las madres jóvenes tienen una probabilidad más elevada de haber rechazado ofertas laborales por causa de la maternidad.

Una vez se vinculan al mercado de trabajo, las madres jóvenes son más tendientes a realizar labores independientes, su probabilidad de estar vinculados a un trabajo asalariado es de alrededor 6 p.p. más baja.

Sin embargo, las características de esta vinculación son distintas a las de los hombres, las actividades como cuenta propia para las mujeres pueden generar horarios más flexibles y autonomía para determinar cuántas horas trabajar.

Cuadro 8.2 Impactos a la vinculación laboral de paternidad/maternidad temprana. Resultados de las estimaciones hacen referencia al ATT, para una muestra de padres ocupados no migrantes entre 25 y 35 años. (Primer empleo)

\begin{tabular}{|c|c|c|c|c|c|c|}
\hline \multirow[b]{3}{*}{ Log Ingreso laboral+ } & \multicolumn{6}{|c|}{ ATT } \\
\hline & \multicolumn{2}{|c|}{ TOTAL } & \multicolumn{2}{|c|}{ Mujeres } & \multicolumn{2}{|c|}{ Hombres } \\
\hline & $-.12619 * * *$ & $(.02190)$ & $-.1374 * * *$ & $(.02734)$ & $-.05488 * *$ & $(.03953)$ \\
\hline Trabaja menos 40 horas & $.02945 * * *$ & $(.0119)$ & $.03710 * * *$ & $(.00713)$ & $.00530 *$ & $(.00661)$ \\
\hline $\begin{array}{c}\text { Trabaja menos de } 28 \\
\text { horas }\end{array}$ & $.02434 * * *$ & $(.0104)$ & $.03470 * * *$ & $(.00523)$ & .00119 & $(.00948)$ \\
\hline Trabaja más de 48 horas & $-.00890 * * *$ & $(.00765)$ & -.00566 & $(.01288)$ & $-.00848 * * *$ & $(.00723)$ \\
\hline $\begin{array}{c}\text { Continuidad trabajo } \\
\text { actual+ }\end{array}$ & .37429 & $(1,9974)$ & $-3,2607 * *$ & $(1,3758)$ & $7,321 * * *$ & $(2,6605)$ \\
\hline $\begin{array}{c}\text { Rechazo ofertas } \\
\text { laborales }\end{array}$ & $.0216 * *$ & $(.00850)$ & $.03012 *$ & $(.01244)$ & .01258 & $(.00679)$ \\
\hline $\begin{array}{l}\text { Trabajo menos de } 12 \\
\text { meses en el último año }\end{array}$ & .00122 & $(.00864)$ & .00400 & $(.01151)$ & $-.00087 * *$ & $(.00340)$ \\
\hline $\begin{array}{c}\text { Trabaja menos de } 40 \text { por } \\
\text { que se ajusta a sus } \\
\text { necesidades }\end{array}$ & $.01353 * * *$ & $(.01099)$ & $.01518 * *$ & $(.01598)$ & .00150 & $(.01097)$ \\
\hline Trabajo secundario & $.00432 * *$ & $(.00453)$ & .00751 & $(.00835)$ & -.00004 & $(.0062)$ \\
\hline Informal (DANE) & $.08356 * *$ & $(.00391)$ & $.0800 * * *$ & $(.00848)$ & $.0632 * * *$ & $(.02032)$ \\
\hline
\end{tabular}




\begin{tabular}{ccccccc} 
Cotiza salud y pensión & $-.09810 * * *$ & $(.01210)$ & $-.09977 * * *$ & $(.01191)$ & $-.0699 * * *$ & $(.01886)$ \\
\hline Independiente & $.06311 * * *$ & $(.01863)$ & $.05077 * * *$ & $(.01354)$ & $.06157 * * *$ & $(.01738)$ \\
\hline Asalariado & $-.06852 * * *$ & $(.00759)$ & $-.067710 * * *$ & $(.01581)$ & $-.04742 * *$ & $(.01503)$ \\
\hline $\begin{array}{c}\text { Subempleado por horas } \\
\begin{array}{c}\text { Subempleado por } \\
\text { competencias }\end{array}\end{array}$ & .00650 & $(.00572)$ & .01048 & $(.00609)$ & -.0008 & $(.00478)$ \\
\hline
\end{tabular}

Errores estándar en paréntesis; Resultados estimados a través de emparejamiento Kernel (Epanechnikov) con ancho de banda 0.05 . Variables dependientes son dicótomas. + representa una variable continua.

Las madres jóvenes buscarían este tipo de vinculación porque requieren de flexibilidades para suplir la demanda de tiempo de sus hijos, en efecto, estas madres tienen una probabilidad de 3,7 p.p. mayor de trabajar medio tiempo y son más propensas a trabajar menos de 40 horas, explicado porque es la jornada que se ajusta a sus necesidades. ${ }^{32}$ En general, las actividades que realizan las madres jóvenes son 9 p.p. más asociadas a actividades informales. Bajo el mismo marco, estas primeras incursiones marcarían un elemento fundamental en la explicación de su trabajo e ingreso en el mediano plazo y no solo estarían atribuidas a la acumulación de capital humano.

No se encontró evidencia de que existan impactos determinantes desde el punto de vista de la estabilidad laboral, o más bien, sobre cambios recurrentes en la ocupación, esto sugiere que es muy factible de que una vez los padres jóvenes se insertan al mercado laboral se perpetúan en estas actividades, al menos en el mediano plazo.

En síntesis, los canales de transmisión de la maternidad/paternidad joven parten de un mismo hecho, el abandono y la no continuidad en la formación de capital humano. Esto viene seguido de una vinculación laboral deteriorada, por sexo, las características de esta primera incursión son sustancialmente distintas, pero problemáticas para ambos casos. Los padres jóvenes en el mediano plazo no están más interesados por buscar o cambiar de empleo, a pesar de que tienen menos ingreso ${ }^{33}$. Las restricciones y los altos costos de un cambio de empleo pueden jugar un papel fundamental en la explicación de esta situación.

Finalmente, un elemento interesante es que los padres jóvenes tienen una probabilidad 75 p.p. más alta de haber sido padres de un segundo hijo, cuando tienen entre 25 y 35 años. La presencia de un segundo hijo indiscutiblemente afecta los ingresos (Olarte \& peña 2010),

\footnotetext{
${ }^{32}$ Para esta estimación se usó la pregunta: ¿Cuál es la razón por la que trabaja menos de 40 horas a la semana?.

${ }^{33}$ No se encontraron diferencias significativas en el subempleo objetivo por horas o competencias o en la búsqueda de empleo.
} 
más aún, si este es concebido siendo también padre joven, como se vio en los resultados del segundo modelo. Un hijo adicional estaría relacionado con los ingresos en la misma vía explicada previamente, acrecentando la probabilidad del abandono escolar y produciendo cambios en la vinculación laboral como resultado de la impaciencia o la búsqueda de actividades más flexibles y compatibles con el cuidado de los hijos. Además, el mantenimiento económico de un segundo hijo haría más fuertes las restricciones para cambiar de ocupación.

\section{Conclusiones y recomendaciones.}

Este estudio constituye uno de los primeros abordajes sobre la baja edad promedio, con respecto a los países desarrollados, en la que los colombianos tienen a sus primogénitos. Tomando una muestra de padres, entre 25 y 35 años, se comprueba que los padres jóvenes enfrentan una penalidad en los ingresos cercana al 11,7\% frente a los padres que postergan su paternidad. El impacto es sustancialmente mayor para las mujeres (12,6\%). Para los hombres el impacto es del orden del 5\%. Los efectos adversos de tener un hijo a edad temprana no solo se explican por ser padre adolescente. Este evento se produce en un momento crítico del ciclo de vida, donde aumenta la probabilidad de interrumpir eventos importantes en la formación de capital humano y genera cambios en la vinculación laboral como consecuencia del cuidado y el mantenimiento económico del niño. Los hombres padres jóvenes son más impacientes para ingresar al mercado laboral, y las mujeres madres jóvenes buscan actividades con mayor flexibilidad. En ambos casos se demuestra deterioro en sus primeras vinculaciones laborales que no se mitigan en el mediano plazo.

Se evidencia que la acumulación de capital humano no es la única vía por la cual se puede explicar la penalidad. Los efectos encontrados no son producto de la tenencia de hijos, en general, pero ser padre a edad temprana conduce a generar condiciones inadecuadas para vincularse al mercado de trabajo, lo que explica en buena medida el efecto sobre los ingresos.

Las políticas públicas para atender esta problemática se encuentran focalizadas en la reducción del embarazo adolescente, es necesario redimensionar los alcances de las 
mismas, porque los resultados negativos de una paternidad temprana pueden llegar a ser más importantes. Esto desde el punto de vista de los ingresos.

Existe evidencia que la baja edad de procreación del primogénito de los colombianos no obedece a situaciones accidentales, sino que está más relacionado a una conciencia cultural e idiosincrática, en la que se asume por parte de los jóvenes que estas edades son las más idóneas u óptimas para ser padres. Por tanto, los lineamientos de política deben buscar en primera instancia incentivar y concienciar a los jóvenes para que posterguen su paternidad. Estas promociones deben tener como objetivo a la población estudiantil, y deben ser instauradas hasta en las instituciones de educación superior.

Se deben promocionar políticas para que los padres jóvenes tengan menores perdidas en términos educativos, puesto que la maternidad temprana está afectando de manera directa la conclusión de logros educativos. Además se deben fomentar programas y estudios que ayuden a impedir una inserción apresurada al mercado de trabajo, puesto que la impaciencia y la búsqueda de flexibilidad, en un mercado laboral caracterizado por tener una elevada tasa de desocupación y alto tiempo de búsqueda para los jóvenes, se convierten en trampas que determinarán los salarios de los jóvenes, al menos en el mediano plazo.

Para el caso de las mujeres, deben evitar tanto la pérdida en términos de educación como la perdida en términos de experiencia laboral, para esto, la formalización laboral juega un rol importante, pues es solo a través de esta se hace factible que las legislaciones que persigan proteger a las madres jóvenes tengan efectos certeros.

Las estimaciones y resultados de este estudio, presentan ciertas limitaciones, principalmente los efectos medidos no incluyen características no observables como la responsabilidad, la impaciencia, o el deseo por los hijos, que pueden ser determinantes tanto de la paternidad temprana como de los ingresos. El método utilizado, tal vez no puede lograr corregir la totalidad del sesgo por no controlar por variables no observables. Pero dada la disponibilidad de datos, ofrece una buena aproximación. Aun así, la definición de padre joven que en el documento se usa, no caracteriza a una población notoriamente marginada o especifica. Por el contrario, caracteriza casi a la mitad de los padres colombianos. Esto ayuda a evitar que los sesgos sean profundos. 
En esta misma línea, la selección de los grupos de tratados y controles se centró en evaluar el promedio de procreación del primogénito de los colombianos, los resultados de las estimaciones podrían variar de acuerdo a los grupos de control escogidos, se sugiere profundizar el tema con un estudio que investigue el beneficio marginal del aplazamiento la paternidad/maternidad por año adicional.

Por otra parte, la muestra seleccionada, al ser población no migrante, hace que se pierda algún grado de validez externa de los resultados. Lo mismo sucede cuando se seleccionan individuos en su primer empleo para hacer el análisis de la vinculación laboral.

La disponibilidad de variables dificultó testear la hipótesis sobre la calidad de las reinserciones escolares de los padres que abandonaron sus estudios de forma temporal por razones de paternidad/maternidad.

La estimación por cortes transversales, y en particular, por solo contar con año de información no permiten estimar si los impactos observados se prolongan o disipan en el largo plazo. Esto podría testearse una vez estén disponibles datos longitudinales en Colombia, o cuando exista una acumulación de cortes transversales suficiente para implementar técnicas de pseudo panel. De esta manera se podría obtener una visión completa de la penalidad de la paternidad/maternidad joven en el curso de la vida. En futuros trabajos sería interesante evaluar también los impactos de ser padre joven en los niveles de empleo, la historia conyugal, la acumulación de activos y beneficios pensionales. 


\section{Bibliografía}

Alcázar L. (2006). "Consecuencias socioeconómicas de la maternidad adolecente, constituye un obstáculo para la formación de capital humano y acceso a mejores empleos?". Centro de investigación y desarrollo INEI, Perú.

Azevedo J. et al. (2012) "Is the baby to blame? An inquiry into de consequences of early childbearing". Policy research working paper 6074. The world bank. Poverty reduction and economic management unit.

Becker G. (1960) An economic analysis of fertility. Demographic and economic Change in developed countries. Princeton: Princeton University press.

Becker G. (1985) "Human capital, effort, and the sexual division of labor" Journal of labor economics, vol 3. No.1 University of Chicago Press. pp 33-58.

Becker G. (1991) "Treatise on the family". Cambridge: Harvard University Press.

Bernal R. \& Peña X. (2011) "Guía práctica para la evaluación de impacto" Centro de Estudios sobre Desarrollo Económico, CEDE. Ediciones Uniandes.

Berthelon M. et al (2010). "Education consequences of adolecent motherhood in Chile". Edición online: politicaspublicas.udp.cl/media/.../Diana_Kruger.p df

Brunner \& Kuhn (2010) "The Impact of Labor Market Entry Conditions on Initial Job Assignment, Human Capital Accumulation, and Wages" IZA Discussion Paper No. 5360.

Chevalier A. \& Viitanen T. (2002) "The long run labour market consequences of teenage motherhood in Britain". Institute for the study of social change, Univerity College Dublin. J popul econ (2003) 16:323-343.

Denise \& Pol (1982) "The individual economic cost of teenage childbearing" Family relations. Vol 31. No 2 pp 249-259.

East P. (1999) "The first teenage pregnacy in the family: does it affect mother's parenting, attitudes, or mother-adolescent communication?" J of marriage and the family ; No 61 (2): 306-319.
Flórez, C. (2000), "Las transformaciones sociodemográficas en Colombia durante el siglo XX”. Bogotá D.C.: Tercer Mundo Editores.

Flórez, C. \& Núñez, J. (2002). Teenage childbearing in Latin American countries. Documento CEDE núm. 1, Bogotá, D.C.: Centro de Estudios sobre de Desarrollo Económico. pp. $1-83$.

Flórez \& Soto (2007) "fecundidad adolecente y pobreza. Diagnóstico y lineamientos de política. Bogotá: Departamento Nacional de Planeación (MERPD).

Gamboa L. \& Zuluaga B., 2013. "Is There a Motherhood Penalty? Decomposing the Family Wage Gap in Colombia," Journal of Family and Economic Issues, Springer, vol. 34(4), pages 421434 ,

Geronimus, A. \& Korenman S. (1992) "The socioeconomic consequences of teen childbearing reconsidered" Quarterly journal of economics 107. No.4 pp 1187-1214.

Gerstenbluth M. et al. (2009) "Impacto de la maternidad adolecente en los logros educativos". Departamento de economía, Facultad de ciencias sociales, Universidad de la república. Documentos de trabajo No. 05/09.

Godfrey, L.G. (1988), "Misspecification tests in econometrics", Cambridge, UK: Cambridge ISBN 0-521-26616-5.

Gutierrez D. (2008) "Efecto de la fecundidad sobre en ingreso laboral femenino" tesis PEG, Bogotá, Universidad de los Andes, Facultad de economía. $40 \mathrm{pp}$.

Hamilton B. (2000) Does Entrepreneurship Pay? An Empirical Analysis of the Returns to SelfEmployment. Journal of Political Economy Vol. 108, No. 3, pp. 604-631.

Heckman, J. (1979). "Sample Selection Bias as a specification error". Econometrica, No 7 Vol. 1: 153-161.

Hotz, V. Joseph, Susan McElroy, \& Seth Sanders (1995) "Assessing the effects of teenage childbearing on maternal outcomes in the U.S: Exploiting a very natural experiment" CarnegieMellon University 
Hotz, V. Joseph, Susan McElroy, \& Seth Sanders (2005) "Teenage childbearing and its life cycle consequences: exploting a natural experiment" Journal of human reources 40(3): 683-715.

Jencks C. (1989) "what is the underclass- and is it growing?" Focus (special issue: defining and measuring the underclass, XII, 14-29.

Joan R. Kahn \& Kay E. Anderson (1992) "Intergenerational Patterns of Teenage Fertility" Demography

Vol. 29, No. 1 pp. 39-57.

Kahn, L. B. (2010). The Long-Term Labor Market Consequences of Graduating from College in a Bad Economy. Labour Economics, 17(2), 303316.

Klepinger et. al (1997) "How does adolescent fertility affect the human capital and wages of young woman" Institute for research on poverty. Discussion paper no. 1145-97.

Madrigal, J. (1984) "Metodología y construcción de un indicador del ingreso familiar. Asociación demográfica costarricense.

Maureen A. (1996) "The education and labor market outcomes of adolescent fathers" Youth society. no. 28:236.

Meade, Christina S.; Kershaw, Trace S.; Ickovics, Jeannette R. (2008) "The intergenerational cycle of teenage motherhood: An ecological approach." Health Psychology, Vol 27(4), 419-429

Mincer, J. (1974) "Schooling, Experience and earning" National bureau of economic research. Journal of Political Economy Vol. 83, No. 2 (Apr., 1975), pp. 444-446.

Moffit R. (1984) "Profiles of Fertility, Labour Supply and Wages of Married Women: A Complete Life-Cycle Model" The review of economic studies. Vol. 51, no. 2. pp 263-278.

Núñez \& Cuesta (2006). "Demografía y pobreza en Colombia". Documento CEDE 2006-30. Issn 1657-7191(edición electrónica).
Núñez (2008) "El efecto del embarazo en la adolescencia sobre el número promedio de años de instrucción de la población." Documentos de Investigación del Proyecto LA5P201. Documento 11.

Olarte L. \& Peña X. 2010 "El efecto de la maternidad sobre los ingresos femeninos" Ensayos sobre política económica vol.28, no.63 pp.190-231.

Oreopoulos P. et al (2006) "the short-and longterm career effects of graduating in a recession: hysteresis and heterogeneity in the market for college graduates" NATIONAL BUREAU OF ECONOMIC RESEARCH. Working Paper 12159 http://www.nber.org/papers/w12159

Oyer, P. (2006). Initial Labor Market Conditions and Long-Term Outcomes for Economists. The Journal of Economic Perspectives, 20(3), 143160.

Silva A., González P. \& Torres M. (2008) "Consideraciones teóricas y empíricas acerca de la fecundidad adolecente". Revista facultad ciencias económica Universidad Nueva Granada. Vol. 16. No 2. pp 47-59.

Taniguchi H. (1999) "The timing of childbearing and women's wages". Vol. 61. No 4. pp 10081019.

Trussell J. (1999) "Economic consequences of teenage childbearing". Family planning perspectives, Guttmacher Institute. Vol.8 No.4. pp 184-190.

Uunk W. (2005) "The impact of young Children on Women's labour supply". Acta sociologica. Sage. Vol. 48 No. 1. pp 41-62.

Trussell J.(1980) "teenage mothers, labour force participation, and wage rates" Canadian studies in population 7:33-48.

Urdinola P. \& Ospino C. (2013) Working paper "Having kids is not good for kids or babies. The Colombian case".

Waldfogel, J 1997 The effects of Children on women's wages American sociological review, vol 62. no.2. 


\section{APENDICE 1. ESTADÍSTICAS DESCRIPTIVAS}

Gráfica 1.1 Rango de edad en que tuvo sus hijos, según número de hijos.

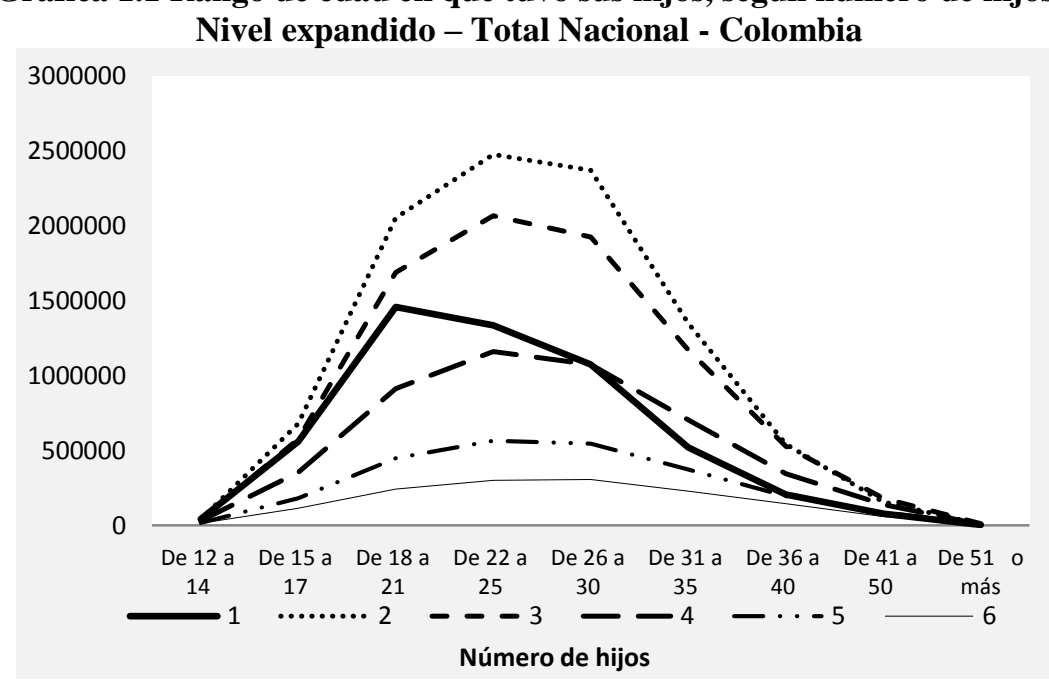

Fuente DANE: Cálculos propios

Gráfica 1.2 Distribución del logaritmo del salario según sexo Total Nacional - datos expandidos 34

(Octubre 2012 - Septiembre 2013)
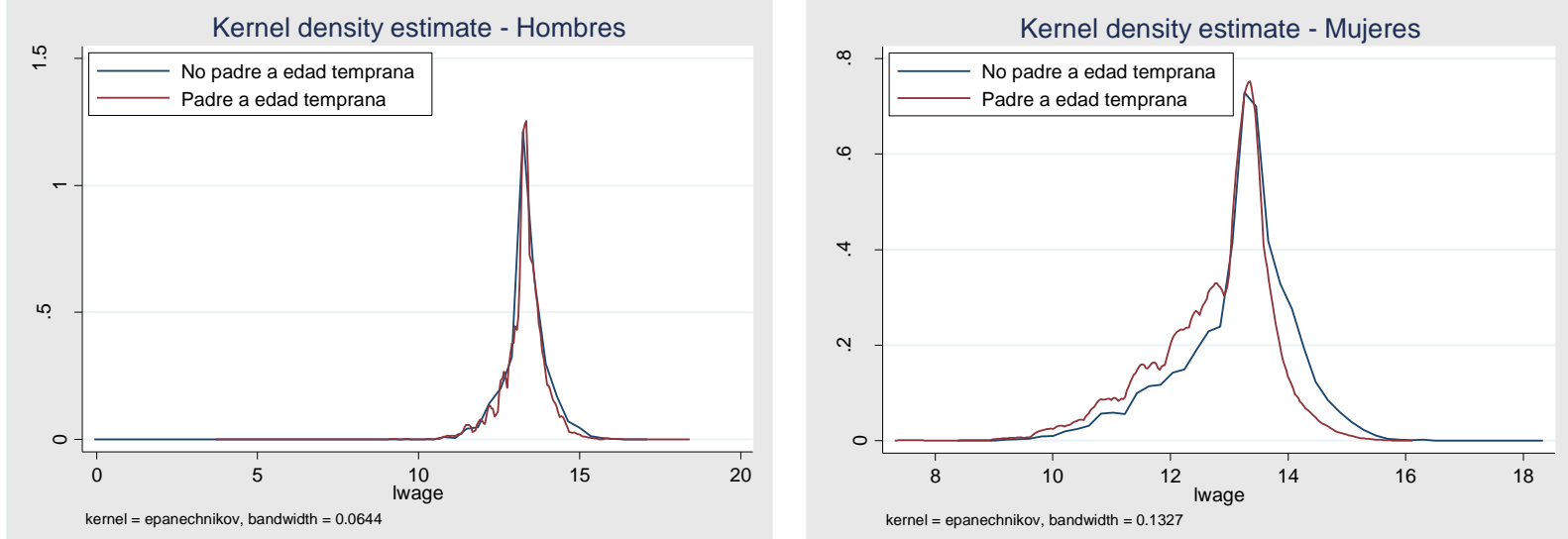

Fuente DANE: Cálculos propios

\footnotetext{
${ }^{34}$ Esta información es calculada para la población objetivo del estudio, es decir, padres entre 25 y 35 años no migrantes. El factor de expansión es recalibrado para ser representativo de doce meses.
} 
Cuadrol.1 Estadísticas descriptivas de las variables involucradas en el cálculo del Propensity Score Matching

\begin{tabular}{|c|c|c|c|c|c|c|c|c|c|}
\hline & \multicolumn{3}{|c|}{ Total } & \multicolumn{3}{|c|}{ Mujeres } & \multicolumn{3}{|c|}{ Hombres } \\
\hline & $\begin{array}{c}\text { No padres a } \\
\text { edad } \\
\text { temprana } \\
\end{array}$ & $\begin{array}{c}\text { Padres a } \\
\text { edad } \\
\text { temprana } \\
\end{array}$ & $\begin{array}{c}\text { Diferencia } \\
\text { de medias } \\
(\mathrm{t}-\mathrm{test})\end{array}$ & $\begin{array}{c}\text { No padres a } \\
\text { edad } \\
\text { temprana }\end{array}$ & $\begin{array}{c}\text { Padres a } \\
\text { edad } \\
\text { temprana } \\
\end{array}$ & $\begin{array}{c}\text { Diferencia } \\
\text { de medias } \\
\text { (t-test) }\end{array}$ & $\begin{array}{c}\text { No padres a } \\
\text { edad } \\
\text { temprana } \\
\end{array}$ & $\begin{array}{c}\text { Padres a } \\
\text { edad } \\
\text { temprana } \\
\end{array}$ & $\begin{array}{c}\text { Diferencia } \\
\text { de medias } \\
\text { (t-test) }\end{array}$ \\
\hline Sexo & 0.480 & 0.720 & $-0.240 * * *$ & 1.000 & 1.000 & 0.000 & 0.000 & 0.000 & 0.000 \\
\hline Edad & 30.541 & 29.880 & $0.661 * * *$ & 30.479 & 29.869 & $0.610 * * *$ & 30.599 & 29.907 & $0.691 * * *$ \\
\hline \multicolumn{10}{|l|}{ Estrato } \\
\hline Bajo & 0.714 & 0.789 & $-0.075 * * *$ & 0.686 & 0.791 & $-0.105^{* * *}$ & 0.740 & 0.783 & $-0.044 * * *$ \\
\hline Medio & 0.226 & 0.148 & $0.078 * * *$ & 0.259 & 0.148 & $0.111 * * *$ & 0.195 & 0.146 & $0.049 * * *$ \\
\hline \multicolumn{10}{|l|}{ Fecundidad municipio } \\
\hline Tasa de fec. adolescente & 21.837 & 22.334 & $-0.497 * * *$ & 21.685 & 22.378 & $-0.693 * * *$ & 21.978 & 22.220 & $-0.243 * * *$ \\
\hline $\begin{array}{l}\text { Fecundidad hogar } \\
1 \text { persona en el hogar } \\
\text { mayor y padre a edad } \\
\text { temprana. } \\
2 \text { personas en el hogar } \\
\text { mayores y padres a edad } \\
\text { temprana. }\end{array}$ & 0.030 & 0.033 & $-0.072 * * *$ & 0.028 & 0.030 & $-0.080 * * *$ & 0.033 & 0.042 & $\begin{array}{l}-0.061 * * * \\
-0.009 * * *\end{array}$ \\
\hline $\begin{array}{l}3 \text { o más personas en el } \\
\text { hogar mayores y padres } \\
\text { a edad temprana. } \\
\text { Educación jefe de } \\
\text { hogar }\end{array}$ & 0.002 & 0.005 & $-0.003 * * *$ & 0.002 & 0.005 & $-0.003 * * *$ & 0.003 & 0.006 & $-0.004 * * *$ \\
\hline Primaria incompleta & 0.052 & 0.085 & $-0.033 * * *$ & 0.033 & 0.083 & $-0.050 * * *$ & 0.070 & 0.091 & $-0.021 * * *$ \\
\hline Superior completa & 0.159 & 0.048 & $0.112 * * *$ & 0.210 & 0.050 & $0.160 * * *$ & 0.113 & 0.041 & $0.071^{* * *}$ \\
\hline Secundaria completa & 0.367 & 0.356 & $0.011 * * *$ & 0.349 & 0.358 & -0.009 & 0.383 & 0.350 & $0.033 * * *$ \\
\hline \multicolumn{10}{|l|}{ Ciudad } \\
\hline Bogotá & 0.038 & 0.043 & $-0.005 * * *$ & 0.036 & 0.040 & $-0.004^{*}$ & 0.039 & 0.051 & $-0.011^{* * *}$ \\
\hline Tunja & 0.029 & 0.027 & 0.002 & 0.032 & 0.026 & $0.006^{* * *}$ & 0.026 & 0.030 & $-0.004^{*}$ \\
\hline Pasto & 0.024 & 0.031 & $-0.007 * * *$ & 0.027 & 0.028 & -0.001 & 0.021 & 0.039 & $-0.018 * * *$ \\
\hline Medellín & 0.035 & 0.043 & $-0.009 * * *$ & 0.034 & 0.041 & $-0.007 * * *$ & 0.035 & 0.050 & $-0.015^{* * *}$ \\
\hline Santa Marta & 0.049 & 0.044 & $0.005 * * *$ & 0.050 & 0.044 & $0.007 * * *$ & 0.048 & 0.044 & 0.004 \\
\hline Riohacha & 0.034 & 0.032 & 0.002 & 0.034 & 0.033 & 0.001 & 0.034 & 0.030 & 0.004 \\
\hline Villavicencio & 0.029 & 0.033 & $-0.003 * *$ & 0.030 & 0.034 & $-0.004 * *$ & 0.029 & 0.030 & -0.001 \\
\hline Manizales & 0.031 & 0.034 & $-0.003 * *$ & 0.032 & 0.035 & -0.003 & 0.030 & 0.032 & -0.002 \\
\hline Popayán & 0.038 & 0.036 & 0.001 & 0.043 & 0.037 & $0.007 * * *$ & 0.033 & 0.036 & -0.003 \\
\hline Ibagué & 0.022 & 0.023 & -0.001 & 0.024 & 0.023 & 0.001 & 0.020 & 0.023 & -0.003 \\
\hline Valledupar & 0.041 & 0.038 & $0.003 *$ & 0.043 & 0.037 & $0.006^{* * *}$ & 0.040 & 0.041 & -0.001 \\
\hline Armenia & 0.021 & 0.025 & $-0.004 * * *$ & 0.021 & 0.026 & $-0.005^{* * *}$ & 0.021 & 0.023 & -0.002 \\
\hline Cali & 0.032 & 0.033 & -0.001 & 0.032 & 0.032 & 0.001 & 0.031 & 0.037 & $-0.006^{* *}$ \\
\hline Bucaramanga & 0.019 & 0.017 & 0.002 & 0.019 & 0.017 & 0.002 & 0.019 & 0.019 & 0.001 \\
\hline Neiva & 0.028 & 0.028 & -0.000 & 0.029 & 0.029 & -0.000 & 0.027 & 0.026 & 0.001 \\
\hline Pereira & 0.023 & 0.028 & $-0.005 * * *$ & 0.022 & 0.028 & $-0.006 * * *$ & 0.024 & 0.027 & -0.003 \\
\hline Dominio Resto & 0.104 & 0.126 & $-0.022 * * *$ & 0.320 & 0.328 & -0.009 & 0.203 & 0.222 & $-0.020 * * *$ \\
\hline Observaciones & 24916 & 25635 & & 11962 & 18453 & & & 12954 & 7182 \\
\hline
\end{tabular}


Cuadro1.2 Estadísticas descriptivas de las variables involucradas en el modelo M.C.O y máxima verosimilitud.

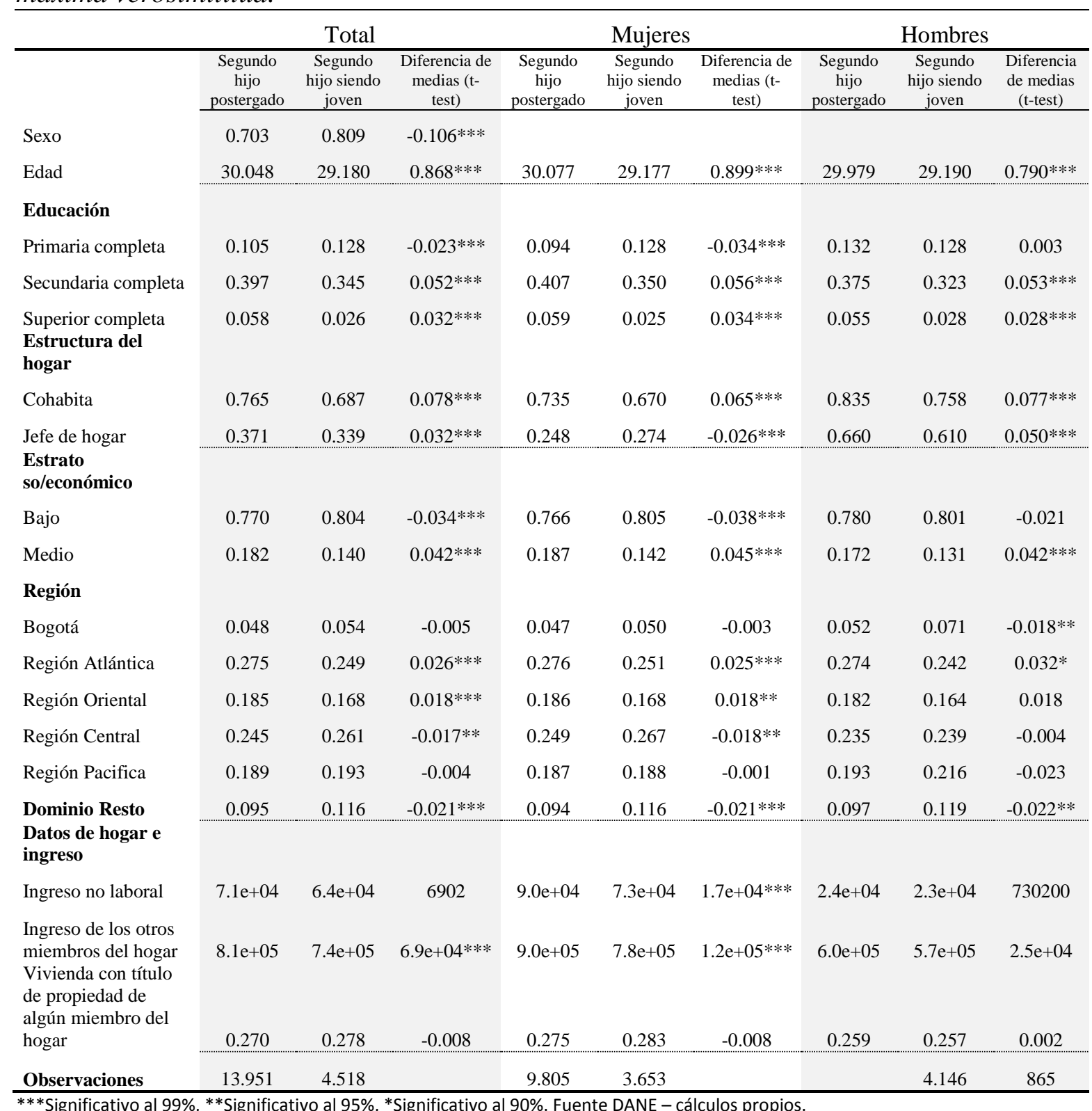




\section{APENDICE 2. METODOS DE EMPAREJAMIENTO, BALANCE Y SOPORTE COMÚN DEL (ATT) PROPENSITY SCORE MATCHING}

Cuadro 2.1 Efecto de la maternidad o paternidad temprana sobre los ingresos laborales. Resultados de las estimaciones por distintos métodos de emparejamiento hacen referencia al ATT, para una muestra de no migrantes entre 25 y 35 años.

\begin{tabular}{|c|c|c|c|c|c|c|c|c|c|c|c|c|}
\hline \multirow{2}{*}{ OLS Regresion } & \multicolumn{12}{|c|}{ Logaritmo del ingreso laboral mensual por hora (Lynhr) } \\
\hline & \multicolumn{3}{|c|}{$\begin{array}{l}\text { Padres a edad temprana en el total de padres } \\
\text { (1) }\end{array}$} & \multicolumn{3}{|c|}{$\begin{array}{l}\text { Padres a edad temprana en el total de } \\
\text { ocupados (padres y no padres) (2) }\end{array}$} & \multicolumn{3}{|c|}{$\begin{array}{l}\text { Padres a edad temprana frente a no } \\
\text { padres (3) }\end{array}$} & \multicolumn{3}{|c|}{$\begin{array}{l}\text { Padres a edad temprana frente padres } \\
\text { con un solo hijo (4) }\end{array}$} \\
\hline Simple & & $\begin{array}{l}-0.2613 \\
(0.0084)\end{array}$ & & & $\begin{array}{l}-0.3160 \\
(0.0075)\end{array}$ & & & $\begin{array}{l}-0.3688 \\
(0.0089)\end{array}$ & & & $\begin{array}{l}-0.17651 \\
(0.01358)\end{array}$ & \\
\hline \multicolumn{13}{|l|}{ Propensity Score Matching } \\
\hline & ATT & $\begin{array}{l}\text { Bootstrap } \\
\text { Std. Err. }\end{array}$ & $\mathrm{P}>|\mathrm{z}|$ & ATT & $\begin{array}{l}\text { Bootstrap } \\
\text { Std. Err. }\end{array}$ & $\mathrm{P}>|\mathrm{z}|$ & ATT & $\begin{array}{l}\text { Bootstrap } \\
\text { Std. Err. }\end{array}$ & $\mathrm{P}>|\mathrm{z}|$ & ATT & $\begin{array}{l}\text { Bootstrap } \\
\text { Std. Err. }\end{array}$ & $\mathrm{P}>|\mathrm{z}|$ \\
\hline Neinearest neighbor (1) & -0.1101 & $(0.01438)$ & 0.000 & -0.1191 & $(0.01362)$ & 0.000 & -0.1372 & $(0.01766)$ & 0.000 & -0.0708 & $(0.02094)$ & 0.021 \\
\hline Neinearest neighbor trimming (1) & -0.0913 & $(0.0148)$ & 0.000 & -0.1066 & $(0.01439)$ & 0.000 & -0.1117 & $(0.01701)$ & 0.000 & -0.0871 & $(0.02229)$ & 0.025 \\
\hline Radius caliper $(0,005)$ & -0.1162 & $(0.00974)$ & 0.000 & -0.1232 & $(0.00868)$ & 0.000 & -0.1345 & $(0.01317)$ & 0.000 & -0.0631 & $(0.01496)$ & 0.002 \\
\hline \multirow[t]{2}{*}{ Kernel (Epanechnikov) } & -0.1178 & $(0.0096)$ & 0.000 & -0.1256 & $(.00860)$ & 0.000 & -0.1358 & $(.0108477)$ & 0.000 & -0.0675 & $(.0132786)$ & 0.002 \\
\hline & \multicolumn{3}{|c|}{30157} & \multicolumn{3}{|c|}{44820} & \multicolumn{3}{|c|}{29266} & \multicolumn{3}{|c|}{14198} \\
\hline Treated & \multicolumn{3}{|c|}{15838} & \multicolumn{3}{|c|}{14310} & \multicolumn{3}{|c|}{14315} & \multicolumn{3}{|c|}{4074} \\
\hline Unteatred & \multicolumn{3}{|c|}{14309} & \multicolumn{3}{|c|}{30501} & \multicolumn{3}{|c|}{14947} & \multicolumn{3}{|c|}{10124} \\
\hline
\end{tabular}

Errores estándar en paréntesis 
Cuadro 2.2 Test de balance entre tratados y controles de las estimaciones (ATT) del cuadro 2.1

\section{Pruebas del balance de la muestra de las especificaciones del Cuadro 2.1 bajo distintos metodos de emparejamiento}

\begin{tabular}{|c|c|c|c|c|c|c|c|c|c|c|c|c|c|c|c|c|c|c|c|}
\hline \multicolumn{5}{|c|}{$\begin{array}{l}\text { Padres a edad temprana en el total de padres } \\
\text { (i) }\end{array}$} & \multicolumn{5}{|c|}{$\begin{array}{l}\text { Padres a edad temprana en el total de } \\
\text { ocupados (ii) (padres y no padres) }\end{array}$} & \multicolumn{5}{|c|}{$\begin{array}{l}\text { Padres a edad temprana frente a no padres } \\
\text { (iii) }\end{array}$} & \multicolumn{5}{|c|}{ Padres a edad temprana frente a no padres (iv) } \\
\hline $\begin{array}{l}\text { Pseudo } \\
\text { R2 }\end{array}$ & LR chi2 & $\mathrm{p}>\mathrm{chi} 2$ & $\begin{array}{c}\text { Mean } \\
\text { Bias }\end{array}$ & $\begin{array}{l}\text { Med } \\
\text { Bias }\end{array}$ & $\begin{array}{l}\text { Pseudo } \\
\text { R2 }\end{array}$ & LR chi2 & $\mathrm{p}>$ chi2 & $\begin{array}{c}\text { Mean } \\
\text { Bias }\end{array}$ & $\begin{array}{l}\text { Med } \\
\text { Bias }\end{array}$ & $\begin{array}{l}\text { Pseudo } \\
\text { R2 }\end{array}$ & LR chi2 & $\mathrm{p}>$ chi 2 & $\begin{array}{c}\text { Mean } \\
\text { Bias }\end{array}$ & $\begin{array}{l}\text { Med } \\
\text { Bias }\end{array}$ & $\begin{array}{l}\text { Pseudo } \\
\text { R2 }\end{array}$ & LR chi2 & $\mathrm{p}>$ chi 2 & $\begin{array}{c}\text { Mean } \\
\text { Bias }\end{array}$ & $\begin{array}{l}\text { Med } \\
\text { Bias }\end{array}$ \\
\hline .140 & 3405.02 & .000 & 9.7 & 2.7 & 0.140 & 13405.02 & 0.000 & 9.7 & 2.7 & 0.140 & 13405.02 & 0.000 & 9.7 & 2.7 & 0.140 & 13405.02 & 0.000 & 9.7 & 2.7 \\
\hline
\end{tabular}

Neinearest neighbor (1)

$\begin{array}{lllllllllllllllllllll}\text { Matched } & 0.005 & 205.15 & 0.000 & 2.3 & 1.8 & 0.004 & 176.46 & 0.000 & 2.2 & 1.3 & 0.004 & 158.24 & 0.000 & 2.0 & 1.1 & 0.004 & 50.05 & 0.009 & 2.2 & 2.0\end{array}$

Neinearest neighbor

trimming (1)

Radius caliper $(\mathbf{0 , 0 0 5})$

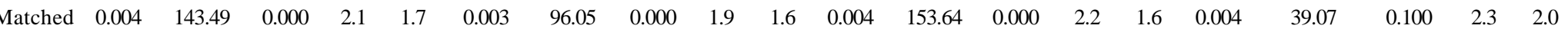

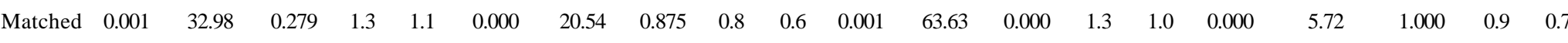

Kernel (Epanechnikov)



Cuadro 2.3 Soporte común de las estimaciones del cuadro 2.1

\begin{tabular}{|c|c|c|c|c|c|c|c|c|c|c|c|c|}
\hline & \multicolumn{12}{|c|}{ Soporte común de las especificaciónes del Cuadro 2.1} \\
\hline & \multicolumn{3}{|c|}{$\begin{array}{l}\text { Padres a edad temprana en el total de padres } \\
\text { (i) }\end{array}$} & \multicolumn{3}{|c|}{$\begin{array}{l}\text { Padres a edad temprana en el total de } \\
\text { ocupados (ii) }\end{array}$} & \multicolumn{3}{|c|}{$\begin{array}{c}\text { Padres a edad temprana frente a no } \\
\text { padres (iii) }\end{array}$} & \multicolumn{3}{|c|}{$\begin{array}{l}\text { Padres a edad temprana frente padres } \\
\text { con un solo hijo (iv) }\end{array}$} \\
\hline & Observations & On support & Off s upport & Observations & On support & Off support & Observations & On support & Off support & Observations & On support & Off support \\
\hline Neinearest neighbor (1) & 33509 & 33499 & 10 & 49292 & 49282 & 10 & 31986 & 319766 & 10 & 15325 & 15318 & 7 \\
\hline Neinearest neighbor trimming (1) & 33509 & 30335 & 3174 & 49292 & 46118 & 3174 & 31986 & 28812 & 3174 & 15325 & 14457 & 868 \\
\hline Radius caliper $(0,005)$ & 33509 & 33493 & 16 & 49292 & 49281 & 11 & 31986 & 319766 & 10 & 15325 & 15316 & 9 \\
\hline Kernel (Epanechnikov) & 33509 & 33499 & 10 & 49292 & 49285 & 7 & 31986 & 319766 & 10 & 15325 & 15318 & 7 \\
\hline
\end{tabular}


Cuadro 2.4 Test de balance entre tratados y controles de las estimaciones (ATT) del cuadro 5.

\begin{tabular}{|c|c|c|c|c|c|c|c|c|c|c|}
\hline & \multicolumn{10}{|c|}{ Pruebas del balance de la muestra de las especificaciones del Cuadro 5.} \\
\hline & \multicolumn{5}{|c|}{ Hombres } & \multicolumn{5}{|c|}{ Mujeres } \\
\hline & $\begin{array}{c}\text { Pseudo } \\
\text { R2 } \\
\end{array}$ & LR chi2 & $\mathrm{p}>$ chi2 & $\begin{array}{c}\text { Mean } \\
\text { Bias } \\
\end{array}$ & $\begin{array}{l}\text { Med } \\
\text { Bias } \\
\end{array}$ & $\begin{array}{c}\text { Pseudo } \\
\text { R2 } \\
\end{array}$ & LR chi2 & p>chi2 & $\begin{array}{c}\text { Mean } \\
\text { Bias } \\
\end{array}$ & $\begin{array}{l}\text { Med } \\
\text { Bias } \\
\end{array}$ \\
\hline \multicolumn{11}{|l|}{ Kernel (Epanechnik ov) } \\
\hline Matched & 0.000 & 3.18 & 1.000 & 0.4 & 0.3 & 0.000 & 9,02 & 1.000 & 0,6 & 0,4 \\
\hline
\end{tabular}

Cuadro 2.5 Test de balance entre tratados y controles de las estimaciones (ATT) del cuadro 6

\begin{tabular}{|c|c|c|c|c|c|c|c|c|c|c|c|c|c|c|c|c|c|c|c|c|}
\hline & \multicolumn{20}{|c|}{ Pruebas del balance de la muestra de las especificaciones del Cuadro 6.} \\
\hline & \multicolumn{5}{|c|}{$\begin{array}{l}\text { Padres a edad temprana en el total de padres } \\
\text { (i) }\end{array}$} & \multicolumn{5}{|c|}{$\begin{array}{l}\text { Padres a edad temprana en el total de } \\
\text { ocupados (ii) (padres y no padres) }\end{array}$} & \multicolumn{5}{|c|}{$\begin{array}{l}\text { Padres a edad temprana frente a no padres } \\
\text { (iii) }\end{array}$} & \multicolumn{5}{|c|}{ Padres a edad temprana frente a no padres (iv) } \\
\hline & $\begin{array}{c}\text { Pseudo } \\
\text { R2 }\end{array}$ & LR chi2 & $\mathrm{p}>$ chi2 & $\begin{array}{c}\text { Mean } \\
\text { Bias }\end{array}$ & $\begin{array}{l}\text { Med } \\
\text { Bias }\end{array}$ & $\begin{array}{l}\text { Pseudo } \\
\text { R2 }\end{array}$ & LR chi2 & p >chi2 & $\begin{array}{c}\text { Mean } \\
\text { Bias }\end{array}$ & $\begin{array}{l}\text { Med } \\
\text { Bias }\end{array}$ & $\begin{array}{l}\text { Pseudo } \\
\text { R2 }\end{array}$ & LR chi2 & $\mathrm{p}>$ chi2 & $\begin{array}{c}\text { Mean } \\
\text { Bias }\end{array}$ & $\begin{array}{l}\text { Med } \\
\text { Bias }\end{array}$ & $\begin{array}{c}\text { Pseudo } \\
\text { R2 }\end{array}$ & LR chi2 & p >chi2 & $\begin{array}{c}\text { Mean } \\
\text { Bias }\end{array}$ & $\begin{array}{l}\text { Med } \\
\text { Bias }\end{array}$ \\
\hline \multicolumn{21}{|l|}{ Kernel (Epanechnikov) } \\
\hline Matched & 0.000 & 7,08 & 1 & 0,6 & 0,4 & 0.000 & 11,14 & 1 & 0,6 & 0,4 & 0,001 & 32,63 & 0,293 & 1,0 & 0,7 & 0 & 3,57 & 1 & 0,7 & 0,5 \\
\hline
\end{tabular}

Cuadro 2.6 Soporte común de las estimaciones del cuadro 6

Soporte común de las especificaciónes del Cuadro 6.

Padres a edad temprana en el total de padres (i) Padres a edad temprana en el total de ocupados (ii) Padres a edad temprana frente a no padres (iii) Padres a edad temprana frente padres con un solo

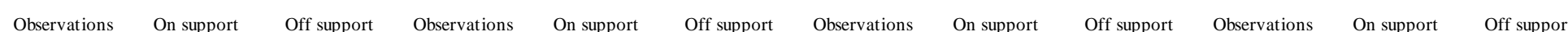

\begin{tabular}{llllllllll} 
Kernel (Epanechnikov) & 28949 & 28946 & 3 & 44732 & 44731 & 1 & 27426 & 27426 & 0 \\
\hline
\end{tabular}




\section{APENDICE 3. PREDICCIÓN DE LA PATERNIDAD JOVEN (P-SCORE)}

Cuadro 3.1 Resultados del modelo Probit, de predicción de la maternidad/paternidad temprana para la construcción del p-score.

\begin{tabular}{|c|c|c|}
\hline VARIABLES & Padre joven & Std Err. \\
\hline Sexo & $0.758 * * *$ & $(0.0130)$ \\
\hline Edad & $-0.0356 * * *$ & $(0.00193)$ \\
\hline Dominio Resto & -0.0113 & $(0.0217)$ \\
\hline Estrato medio & $-0.206 * * *$ & $(0.0287)$ \\
\hline Estrato bajo & 0.0301 & $(0.0251)$ \\
\hline Tasa de fec. Adolescente municipio & $0.0281 * * *$ & $(0.00212)$ \\
\hline $\begin{array}{c}1 \text { persona en el hogar mayor y padre a } \\
\text { edad temprana }\end{array}$ & $0.269 * * *$ & $(0.0148)$ \\
\hline $\begin{array}{c}2 \text { personas en el hogar mayores y } \\
\text { padres a edad temprana }\end{array}$ & $0.155 * * *$ & $(0.0337)$ \\
\hline $\begin{array}{c}3 \text { o más personas en el hogar mayores } \\
\text { y padres a edad temprana }\end{array}$ & $0.598 * * *$ & $(0.103)$ \\
\hline Jefe de Hogar & $0.198 * * *$ & $(0.0137)$ \\
\hline Jefe con secundaria completa & $-0.137 * * *$ & $(0.0129)$ \\
\hline Jefe con primaria incompleta & $0.228 * * *$ & $(0.0248)$ \\
\hline Jefe con superior completa & $-0.829 * * *$ & $(0.0221)$ \\
\hline Tunja & $0.334 * * *$ & $(0.0383)$ \\
\hline Pasto & $0.323 * * *$ & $(0.0363)$ \\
\hline Medellín & $0.299 * * *$ & $(0.0311)$ \\
\hline Santa Marta & $0.156 * * *$ & $(0.0294)$ \\
\hline Bogotá & $0.394 * * *$ & $(0.0319)$ \\
\hline Riohahca & $0.140 * * *$ & $(0.0340)$ \\
\hline Villavicencio & $0.205 * * *$ & $(0.0345)$ \\
\hline Manizales & $0.224 * * *$ & $(0.0342)$ \\
\hline Popayan & $0.145 * * *$ & $(0.0321)$ \\
\hline Ibagué & $0.162 * * *$ & $(0.0397)$ \\
\hline Valledupar & $0.0887 * * *$ & $(0.0309)$ \\
\hline Armenia & $0.197 * * *$ & $(0.0400)$ \\
\hline Cali & $0.175 * * *$ & $(0.0339)$ \\
\hline Bucaramanga & $0.162 * * *$ & $(0.0449)$ \\
\hline Neiva & $0.0974 * * *$ & $(0.0359)$ \\
\hline Pereira & $0.151 * * *$ & $(0.0380)$ \\
\hline Constant & -0.0916 & $(0.0800)$ \\
\hline Observations & 50,551 & \\
\hline
\end{tabular}

Errores estándar en paréntesis $* * * \mathrm{p}<0.01, * * \mathrm{p}<0.05, * \mathrm{p}<0.1$ 
$\underline{\text { Regresiones auxiliares }}$

Cuadro 3.2 Efecto de la maternidad o paternidad temprana sobre los ingresos laborales. Resultados de las estimaciones por distintos métodos de emparejamiento hacen referencia al ATT, por ultimo diploma alcanzado.

\begin{tabular}{|c|c|c|c|}
\hline & \multicolumn{3}{|c|}{ Logaritmo del ingreso laboral mensual por hora (Lynhr) } \\
\hline & \multicolumn{3}{|c|}{ Padres a edad temprana en el total de padres (i) } \\
\hline & Bachiller & Tecnico & Universitario \\
\hline \multicolumn{4}{|l|}{ Propensity Score Matching } \\
\hline Kernel (Epanechnikov) ATT & $-.0272635 * *$ & $-.0233522 *$ & $-.1007655^{* * *}$ \\
\hline Bootstrap Std. Err. & $(.0140335)$ & $(.0212248)$ & $(.016951)$ \\
\hline
\end{tabular}

Errores estándar en paréntesis $* * * \mathrm{p}<0.01, * * \mathrm{p}<0.05, * \mathrm{p}<0.1$

\section{APENDICE 4}

Preguntas utilizadas:

Para la evaluación de los canales de impacto se utilizaron las preguntas de la GEIH:

\begin{tabular}{|c|c|c|c|c|c|}
\hline $\begin{array}{l}\text { ¿En algún momento abandonó sus } \\
\text { estudios por razones de maternidad o } \\
\text { paternidad? }\end{array}$ & 5 & & \begin{tabular}{|l|l|}
1 & $\square$ \\
2 & \\
\end{tabular} & \multicolumn{2}{|l|}{$\begin{array}{l}\text { Pase a } 6 \\
\text { Pase a } 7\end{array}$} \\
\hline $\begin{array}{l}\text { ¿El abandono de sus estudios fue } \\
\text { permanente o temporal? }\end{array}$ & 6 & a. & $\begin{array}{l}\text { Permanente } \\
\text { Temporal }\end{array}$ & \multicolumn{2}{|c|}{  } \\
\hline $\begin{array}{c}\text { ¿En algún momento abandonó su } \\
\text { ocupación o trabajo por razones de } \\
\text { maternidad o paternidad? } \\
\begin{array}{|l}\text { Solo para ocupados, desocupados, cesantes } \\
\text { e inactivos que han trabajado. }\end{array} \\
\end{array}$ & 7 & & \begin{tabular}{l|l|}
1 & \\
2 & \\
\end{tabular} & $\begin{array}{l}\text { Pase a } 8 \\
\text { Pase a } 10\end{array}$ & \\
\hline $\begin{array}{l}\text { ¿El abandono de su ocupación o trabajo } \\
\text { fue permanente o temporal? }\end{array}$ & 8 & a. & $\begin{array}{l}\text { Permanente } \\
\text { Temporal }\end{array}$ & 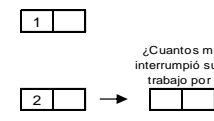 & $\begin{array}{l}\text { es en total } \\
\text { cupación } \\
\text { cuaroin? }\end{array}$ \\
\hline $\begin{array}{l}\text { ¿Cuántas veces ha dejado su trabajo u } \\
\text { ocupación debido a su maternidad o } \\
\text { paternidad? }\end{array}$ & 9 & $\begin{array}{l}\text { a. } \\
\text { b. } \\
\text { c. } \\
\text { d. } \\
\text { e. }\end{array}$ & $\begin{array}{l}\text { Una } \\
\text { Dos } \\
\text { Tres } \\
\text { Cuatro } \\
\text { Cinco o más }\end{array}$ & \begin{tabular}{|l|l}
1 & \\
2 & \\
3 & \\
4 & \\
5 & \\
5 & \\
\end{tabular} & \\
\hline $\begin{array}{l}\text { ¿Ha dejado pasar oportunidades laborales } \\
\text { debido a su maternidad o paternidad? } \\
\text { Pase a } 11 \text { si es inactivo, sí no pase al } \\
\text { capitulo Q }\end{array}$ & 10 & № 2 & $\begin{array}{ll}\square & \rightarrow \\
\text { a. Rech } \\
\text { b. Rech } \\
\text { c. Nop } \\
\text { nego } \\
\text { d. No p } \\
\text { e. Otra } \\
\square\end{array}$ & $\begin{array}{l}\text { Cuál fue la principal } \\
\text { oportunidad: } \\
\text { nazar una oferta laboral } \\
\text { azazar un ascenso } \\
\text { oder abrir o emprender un } \\
\text { cio } \\
\text { oder expandir su negocio } \\
\text { ¿Cuál? }\end{array}$ & \begin{tabular}{|l|l|}
1 & \\
2 & \\
3 & \\
\multicolumn{3}{|l|}{} \\
4 & \\
4 & \\
5 & \\
\end{tabular} \\
\hline
\end{tabular}


$\underline{\text { Bases de datos adicionales: }}$

- Estadísticas vitales EV-DANE - cifras del registro de nacido vivo de estadísticas y el registro civil de nacimiento de la Registraduria Nacional.

Son registros administrativos vitales son estadísticas continuas que recogen información sobre nacimientos, defunciones fetales y no fetales, que permiten contar con información que revela los cambios ocurridos en los niveles y patrones de mortalidad y fecundidad, proporcionando una visión dinámica de la población, como complemento al enfoque estático que proveen los censos.

Uso dentro del documento: Esta base fue utilizada para la predicción de paternidad joven, es un registro administrativo permitió construir una variable de la tasa de fecundidad adolescente por municipio desde el año 1998 hasta el 2012. Esta misma información para antes de 1998 era recolectada a través del registro civil de nacimiento de la Registraduría Nacional. 